\title{
THE CONSISTENT HISTORIES APPROACH TO LOOP QUANTUM COSMOLOGY
}

\author{
David A. Craig \\ Department of Chemistry and Physics, Le Moyne College \\ Syracuse, New York, 13214, USA \\ craigda@lemoyne.edu
}

Received Day Month Year

Revised Day Month Year

\begin{abstract}
We review the application of the consistent (or decoherent) histories formulation of quantum theory to canonical loop quantum cosmology. Conventional quantum theory relies crucially on "measurements" to convert unrealized quantum potentialities into physical outcomes that can be assigned probabilities. In the early universe and other physical contexts in which there are no observers or measuring apparatus (or indeed, in any closed quantum system), what criteria determine which alternative outcomes may be realized and what their probabilities are? In the consistent histories formulation it is the vanishing of interference between the branch wave functions describing alternative histories - as determined by the system's decoherence functional - that determines which alternatives may be assigned probabilities. We describe the consistent histories formulation and how it may be applied to canonical loop quantum cosmology, describing in detail the application to homogeneous and isotropic cosmological models with scalar matter. We show how the theory may be used to make definite physical predictions in the absence of "observers". As an application, we demonstrate how the theory predicts that loop quantum models "bounce" from large volume to large volume, while conventional "Wheeler-DeWitt"-quantized universes are invariably singular. We also briefly indicate the relation to other work.
\end{abstract}

Keywords: loop quantum cosmology; consistent histories; decoherence; alternative formulations of quantum mechanics

PACS numbers: 98.80.Qc,04.60.Pp,03.65.Yz,04.60.Ds,04.60.Kz

\section{Introduction}

The quantum mechanics employed by physicists every day to make predictions concerning the outcomes of experimental measurements is not, as it is conventionally understood, sufficient to make predictions in physical situations in which a clearly defined concept of "measurement" is absent. Prime examples of such physical situations may be found in the early universe, or indeed, any time or place in the universe where measuring (recording) apparatus are not present. How, then, may physical theories offer clear and unambiguous predictions concerning the behavior of the physical universe in the moments surrounding the epoch normally considered to be the big bang? 
One (possibly partial) answer to this question lies in the consistent (or decoherent) histories formulation of quantum theory pioneered and subsequently developed by Griffiths, ${ }^{1}$ Omnes,${ }^{2}$ Gell-Mann and Hartle, ${ }^{3-6}$ Halliwell ${ }^{7-11}$ and others. ${ }^{12-14}$ In this framework quantum theory is supplemented by a consistency condition which must be satisfied in any specific physical situation in order for quantum theory to offer definite predictions concerning that situation. This condition amounts to the requirement that there is essentially no interference (overlap) between the branch wave functions corresponding to each of the alternative quantum histories describing that scenario. Only in that case can quantum theory consistently assign definite probabilities to each of those possible alternative histories. This condition of "decoherence" a or "consistency" among the branch wave functions is satisfied in any physical scenario that would normally be thought of as a classical "measurement situation", ${ }^{\mathrm{b}}$ and hence the framework of consistent histories encompasses the ordinary quantum mechanics of measurements - sans the additional hypothesis of "collapse of the wave function" upon measurement. However, it also extends it in an objective, observer-independent manner to a wide range of physical situations in which observers and measuring apparatus are not present, thus giving quantum theory a voice even when and where physicists themselves do not have one.

The central mathematical object in the consistent histories framework is the decoherence functional. It is a natural generalization of the notion of the quantum state of a system as it arises in the algebraic formulation of quantum theory, and is a sesquilinear functional of the possible quantum histories of the system. The decoherence functional measures the quantum interference between the possible histories, and, when that interference vanishes among all the possible histories i.e. the family of histories decoheres or "is consistent" - the probabilities of each of them. In this way the decoherence functional - the quantum state itself - provides definite quantum predictions for the behavior of a system even in the absence of observers, recording apparatus, or "measurements". c

In this contribution we will summarize recent work on the application of these

\footnotetext{
aThis usage of the term "decoherence" is related to, but conceptually distinct from, the broadly understood phenomenon of environmental decoherence. ${ }^{15-19}$ Physical mechanisms which engender environmental decoherence may thereby lead to the decoherence of the corresponding histories. However, other physical processes such as occur in typical laboratory "measurement situations" may also lead to decoherence of histories in the sense the term is applied here.

${ }^{\mathrm{b}}$ This is epitomized by the two-slit experiment. When the slit through which the particle passes is measured, in the sense that sufficient information is recorded to determine it, the interference between the branch wave functions corresponding to passage through either the upper or lower slit separately is destroyed, and physically meaningful probabilities can be assigned to which slit the particle traversed. Otherwise, the alternative wave functions interfere, and quantum mechanics simply has nothing to say - i.e. cannot assign a probability in a logically consistent manner about through which slit the particle passed.

${ }^{\mathrm{c}}$ What the consistent histories framework does not dictate is which family of quantum histories one interrogates. Different choices of family may provide very different pictures of "what happens". This, indeed, is a manifestation of contextuality in the quantum mechanics of history - quantum mechanics is irreducibly a contextual theory, and thereby paints a contextual picture of reality.
} 
ideas to loop quantum cosmology, developed in collaboration with Parampreet Singh, ${ }^{20-25}$ and based on earlier work of J.B. Hartle and others. ${ }^{5-14}$ All of the discussion will be formulated in the context of a flat, homogeneous and isotropic Friedmann-Lemaitre-Robertson-Walker (FLRW) cosmological model sourced by a massless, minimally coupled scalar field. In section 2 we describe the consistent histories formalism in general terms. We then discuss in section 4 the implementation of this formalism in both a conventional so-called "Wheeler-DeWitt" quantization of this cosmology, as well as a distinct loop quantization of the same model. In section 5 we illustrate the application of this formalism to show how it may be used to make predictions concerning quantum histories of various physical quantities. As an important illustration, we show that in the Wheeler-DeWitt quantization, all quantum states are invariably singular, as they are in the classical theory. By contrast, in the loop quantization all quantum states remain non-singular, and indeed "bounce" from large volume to large volume, approaching well-defined states of the corresponding Wheeler-DeWitt quantization. In this way we illustrate how consistent histories formulations of quantum cosmological theories may be defined and then deployed to make consistent quantum predictions in these theories in the absence of the observers or measurements typically viewed as essential to prediction in quantum mechanics.

\section{Generalized Consistent Histories Quantum Theory}

A "generalized quantum mechanics", as originally defined by Hartle, ${ }^{5,6}$ consists in the specification of four ingredients: (i) The fine-grained histories $h$ of the system, the most refined descriptions of the possible alternative physical histories of the system it is possible to give. In Lagrangian mechanics, for example, for a set of generalized coordinates $\left\{q_{i}\right\}$, the fine-grained histories are all possible paths $\left\{q_{i}(t)\right\}$. The fine-grained histories may be collected into exhaustive (complete) sets of mutually exclusive alternative histories which we will sometimes denote by $\mathcal{S}=\{h\}$. Depending upon the theory, there may be many distinct (possibly incompatible) such exclusive, exhaustive sets of histories, as happens for example in ordinary Hilbert space quantum mechanics because of the existence of non-commuting operators. (Explicit examples of histories in Hilbert space quantum theory will be given below.) (ii) The allowed coarse-grainings (partitions) of exclusive, exhaustive sets of alternative histories into more coarse-grained descriptions of the system. For example, in a diffeomorphism-invariant theory it would be typical to require coarse-grainings to be themselves diffeomorphism-invariant. (It is common to denote by $\bar{h}$ a course-grained history that contains the history $h$ when it is convenient to refer back to $h$ specifically. It is also common to allow $h$ alone to represent both fine-grained and coarse-grained histories as is convenient in context.) It is assumed that all exclusive, exhaustive sets of fine-grained histories $\mathcal{S}=\{h\}$ have a common complete coarse-graining $u=\cup_{h \in \mathcal{S}} h$. (iii) The decoherence functional $d$. The decoherence functional is a complex-valued function on pairs of histories that is 
(i) Hermitian: $\quad d\left(h, h^{\prime}\right)=d\left(h^{\prime}, h\right)^{*}$

(ii) Positive: $\quad d(h, h) \geq 0$

(iii) Additive ("Principle of Superposition"): $\quad d\left(\bar{h}, \bar{h}^{\prime}\right)=\sum_{h \in \bar{h}} \sum_{h^{\prime} \in \overline{h^{\prime}}} d\left(h, h^{\prime}\right)$

(iv) Normalized: $\quad \sum_{h, h^{\prime} \in \mathcal{S}} d\left(h, h^{\prime}\right)=1$

Note these conditions imply $d(u, u)=1$. Finally, the decoherence functional is used to define (iv) the decoherence condition that determines when probabilities may be assigned to the histories in an exclusive, exhaustive set $\mathcal{S}$. The simplest and most common decoherence condition (usually called "medium decoherence" in the literature ${ }^{26,27}$ ) simply requires that the decoherence functional be diagonal on a set of histories $\mathcal{S}$ in order for probabilities to be assigned to the histories in $\mathcal{S}$. In that case the diagonal elements of $d$ are the corresponding probabilities, and $\mathcal{S}$ is said to be a consistent or decoherent set of histories:

$$
d\left(h, h^{\prime}\right)=p(h) \delta_{h, h^{\prime}} \quad \forall h, h^{\prime} \in \mathcal{S}
$$

if $\mathcal{S}$ is a consistent set. Only when $\mathcal{S}$ is consistent may the diagonal elements of $d$ be interpreted as (Kolmogorov) probabilities, for which $p(h) \geq 0$ and $\sum_{h \in \mathcal{S}} p(h)=1$ (whence the term "consistent histories".) In particular, if decoherence does not obtain, the putative "probabilities" $p(h)$ do not add consistently in the sense that $p\left(h_{1}+h_{2}\right) \neq p\left(h_{1}\right)+p\left(h_{2}\right)$, as happens for example in the two-slit experiment when the slit traversed is not measured.

It is the consistency condition (1) that alone determines whether or not probabilities may be meaningfully assigned in any family of histories, and not any notion of "observer" or "measurement" (though it does reproduce the predictions of ordinary Copenhagan quantum theory when quantum measuring apparatus are included in the system.) It is an objective criterion that depends only on the system's quantum state and dynamics - and, of course, on the set of histories in question.

In ordinary Hilbert space quantum theory, fine-grained histories may be specified by sequences of eigenvalues $a_{k_{i}}^{\alpha_{i}}$ of operators $A^{\alpha_{i}}$ at a sequence of times $\left\{t_{i}\right\}: h=$ $\left(a_{k_{1}}^{\alpha_{1}}, a_{k_{2}}^{\alpha_{2}}, \cdots\right){ }^{\mathrm{d}}$ Corresponding to each history $h$ may be defined the operator

$$
C_{h}=P_{a_{k_{1}}}^{\alpha_{1}}\left(t_{1}\right) P_{a_{k_{2}}}^{\alpha_{2}}\left(t_{2}\right) \cdots P_{a_{k_{n}}}^{\alpha_{n}}\left(t_{n}\right),
$$

where if $U(t)$ is the theory's propagator, $P_{a}\left(t_{i}\right)=U\left(t_{i}, t_{0}\right)^{\dagger}|a\rangle\langle a| U\left(t_{i}, t_{0}\right)$ is a Heisenberg picture projection operator. $C_{h}$ is called the "class operator" for the history $h$, and is typically identified with $h$ itself. Coarse-grainings of these histories then correspond to the operator sums

$$
C_{\bar{h}}=\sum_{a_{k_{1}} \in \Delta a_{\bar{k}_{1}}} \sum_{a_{k_{2}} \in \Delta a_{\bar{k}_{2}}} \cdots \sum_{a_{k_{n}} \in \Delta a_{\bar{k}_{n}}} P_{a_{k_{1}}}^{\alpha_{1}}\left(t_{1}\right) P_{a_{k_{2}}}^{\alpha_{2}}\left(t_{2}\right) \cdots P_{a_{k_{n}}}^{\alpha_{n}}\left(t_{n}\right)
$$

\footnotetext{
d To help clarify the confusing but necessary notation, note that the spectrum of the operator $A^{\alpha}$ is $\left\{\cup_{k} a_{k}^{\alpha}\right\}$. In other words, the superscript labels the observable, while the subscript labels the eigenvalues of that observable.
} 
corresponding to the history $\bar{h}=\left(\Delta a_{\bar{k}_{1}}^{\alpha_{1}}, \Delta a_{\bar{k}_{2}}^{\alpha_{2}}, \cdots\right)$ in which the value of $A^{\alpha_{1}}$ is in the range $\Delta a_{\bar{k}_{1}}^{\alpha_{1}}$ labeled by $\bar{k}_{1}$ (so the spectrum of $A^{\alpha}$ is the union of the intervals $\left.\left\{\cup_{k} \Delta a_{k}^{\alpha}\right\}\right)$, and so on. The completely coarse-grained history $u$ then corresponds to

$$
C_{u}=\sum_{h \in \mathcal{S}} C_{h}=\mathbb{1}
$$

The decoherence functional corresponding to ordinary quantum mechanics is

$$
d\left(h, h^{\prime}\right)=\operatorname{tr}\left[C_{h}^{\dagger} \rho C_{h^{\prime}}\right]
$$

where $\rho$ is the initial density matrix. ${ }^{\text {e }}$ If the intial state is pure, $\rho=|\psi\rangle\langle\psi|$, this reduces simply to

$$
d\left(h, h^{\prime}\right)=\left\langle\psi_{h^{\prime}} \mid \psi_{h}\right\rangle
$$

where

$$
\left|\psi_{h}(t)\right\rangle=U\left(t, t_{0}\right) C_{h}^{\dagger}|\psi\rangle
$$

is the so-called "branch wave function" corresponding to the history $h$. Up to normalization, it is simply the wave function at time $t$ a system that began in the state $|\psi\rangle$ would have were the system observed to have had values of observable $A^{\alpha_{1}}$ in $\Delta a_{k_{1}}^{\alpha_{1}}$ at time $t_{1}, A^{\alpha_{2}}$ in $\Delta a_{k_{2}}^{\alpha_{2}}$ at time $t_{2}$, and so on, i.e. for the system to have been observed to "follow" the particular history $h$. However, in consistent histories quantum theory we do not (necessarily) have observers as an essential element of the predictive scheme, and consequent wave function "collapse". Here, it appears simply as a description of one of the many possible alternative histories of the system, and it is up to decoherence to decide if the corresponding family of histories is consistent, and if so, what the probability of each such decohering history may be. ${ }^{\mathrm{f}}$

If there is also a "final condition" $\rho_{\omega}$ in addition to an "initial condition" $\rho_{\alpha}$, then the decoherence functional may be defined similarly by $d\left(h, h^{\prime}\right)=\operatorname{tr}\left[\rho_{\omega} C_{h}^{\dagger} \rho_{\alpha} C_{h^{\prime}}\right]$. If $\rho_{\alpha}=\sum_{i} \alpha_{i}\left|\Psi_{i}\right\rangle\left\langle\Psi_{i}\right|$ and $\rho_{\omega}=\sum_{i} \omega_{i}\left|\Phi_{i}\right\rangle\left\langle\Phi_{i}\right|$, this reduces to

$$
d\left(h, h^{\prime}\right)=\sum_{i, j} \alpha_{i} \omega_{j}\left\langle\Psi_{i}\left|C_{h}\right| \Phi_{j}\right\rangle^{*}\left\langle\Psi_{i}\left|C_{h^{\prime}}\right| \Phi_{j}\right\rangle .
$$

Finally, given a path integral formulation of a quantum theory, if the propagator is given by

$$
U\left(t, t_{0}\right)=\int \delta q e^{i \int_{t_{0}}^{t} d t S[q]}
$$

\footnotetext{
${ }^{\mathrm{e}}$ In this form it should be clear in what sense the decoherence functional is a natural generalization of the notion of the "quantum state" of the system as it arises in the algebraic formulation of quantum theory, to measure interference between histories as well as their probabilities. ${ }^{28,29}$

${ }^{\mathrm{f}}$ See Refs. 22, 24 for some discussion of the leading factor of the propagator $U$, which is not strictly necessary - it cancels in the decoherence functional - and is introduced only in order to enable us to think of $\left|\psi_{h}\right\rangle$ as an evolving solution of the Schrödinger equation for all $t$.
} 
for some action $S[q]$, then class operators may be defined by

$$
C_{h}^{\dagger}\left(t, t_{0}\right)=\int_{q(t) \in h} \delta q e^{i \int_{t_{0}}^{t} d t S[q]} .
$$

In other words, in a path integral formulation, coarse-grained histories are defined by partitions of the space of paths which appear in the path integral. Considerable further detail concerning the construction of the decoherence functional in theories whose amplitudes are defined by path integrals, both relativistic and nonrelativistic, may be found in Refs. 5, 6, 8, 9, 10, 11, 13, 30. In this way, in conjunction with Eq. (8), a consistent histories formulation may be given to spin foam models of quantum gravity ${ }^{31}$ and loop quantum cosmology, ${ }^{30}$ though we will not discuss these here.

\subsection{Prediction in consistent histories quantum theory}

The process of making a physical prediction in this formulation of quantum theory proceeds as follows. Given a quantum state $|\psi\rangle$ and its associated decoherence functional, one constructs the class operators for the family of histories corresponding to the physical question in which one is interested - for example, what (coarse-grained) path does a quantum particle follow through a two-slit apparatus. The decoherence functional is then calculated to determine whether or not that family of histories decoheres i.e. is consistent. If the family is consistent, the decoherence functional determines the probability of each history in the family, and the quantum question has been answered.

However, if the family of histories is not consistent, then no probabilities can be assigned, and quantum mechanics says that the question being asked has no consistent answer within the theory. Familiar examples are replete in ordinary quantum theory - e.g. the precise values of the position and momentum of a particle at the same moment, or which slit a particle passed through in the two slit experiment if no information was gathered to determine it. This important point is perhaps worth some emphasis: the inability of quantum mechanics to make definite predictions concerning what are classically perfectly sensible physical statements is not in any way a unique feature of consistent histories quantum theory. What the consistent histories formulation provides is simply an observer-independent criterion, derived from the quantum state itself, for determining when that is the case, rather than relying on the Copenhagen notion of a wave function-collapsing "measurement", which is in any case impossible to make sense of in environments such as the early universe.

We will see how this scheme works in quantum cosmology in the sequel.

\section{Homogeneous and Isotropic Scalar Cosmologies}

We consider what must be the simplest possible cosmological model, namely, a flat, homogeneous and isotropic Friedmann-Lemaitre-Robertson-Walker cosmology 
sourced by a single massless, minimally coupled scalar field. This model has two relevant advantages. First, it is simple enough that it admits two distinct, exactly solvable quantizations: a conventional "Wheeler-DeWitt" quantization, and a unitarily inequivalent loop quantization, the exactly solvable version of which is dubbed sLQC. Moreover, the loop quantization can be cast in the form of a spin-foam (quasi"path integral") quantization of flat scalar FLRW, with an explicitly solvable vertex expansion. It is thus a perfect playground within which to explore and contrast the consistent histories formulations of each of these three quantizations in a context in which mathematically explicit expressions and exact calculations are possible. Second, in this model the scalar field emerges as an internal physical "clock". While not essential to the consistent histories formulation, the presence of an internal clock variable does make it easier to conceptualize and interpret the results. Thus, this model is rich enough to supply an exceptional proving ground for the exploration of the technical and conceptual issues associated with the consistent histories formulation of quantum gravitational models, in a technically manageable context.

\subsection{Classical scalar cosmologies}

The FLRW metrric for a homogeneous isotropic universe may be expressed as

$$
g_{a b}=-N(t)^{2} d t_{a} d t_{b}+a(t)^{2} \stackrel{\circ}{q}_{a b},
$$

where $a(t)$ is the scale factor, $N(t)$ is the lapse, and $\stackrel{\leftrightarrow}{q}_{a b}$ is a fixed, flat fiducial metric on the spatial slices $\Sigma$, which we take to be topologically $\mathbb{R}^{3}{ }^{32}$ To construct a Hamiltonian formulation of the dynamics spatial integrals over finite volumes are required. Therefore, one introduces a fixed fiducial cell $\mathcal{V}$ with volume $\stackrel{\circ}{V}$ relative to $\stackrel{\circ}{q}_{a b}$, the physical volume of which is therefore $V=a^{3} \stackrel{\circ}{V}$. The Einstein-Hilbert action for a flat $(k=0)$ FLRW universe sourced by a massless, minimally coupled scalar field becomes, after integration over the fiducial spatial volume $\mathcal{V}$,

$$
S=\stackrel{\circ}{V} \int d t\left\{-\frac{3}{8 \pi G} \frac{a \dot{a}^{2}}{N}+\frac{1}{2} a^{3} \frac{\dot{\phi}^{2}}{N}\right\},
$$

where the dot denotes a derivative with respect to $t$. Choosing phase space variables with Poisson brackets $\left\{a, p_{a}\right\}=1$ and $\left\{\phi, p_{\phi}\right\}=1$, the canonical momenta are

$$
p_{a}=-\frac{3}{4 \pi G} \stackrel{\circ}{V} a \frac{\dot{a}}{N}, \quad p_{\phi}=\stackrel{\circ}{V} a^{3} \frac{\dot{\phi}}{N} .
$$

The Hamiltonian is then

$$
H=\frac{1}{\stackrel{\circ}{V}}\left\{-\frac{2 \pi G}{3} \frac{N}{a} p_{a}^{2}+\frac{1}{2} \frac{N}{a^{3}} p_{\phi}^{2}\right\}
$$


For comparison with loop quantum cosmology it turns out to be convenient to make a canonical transformation to a different set of variables, a volume variable ${ }^{g}$

$$
\nu=\varepsilon \frac{1}{2 \pi l_{p}^{2}} \frac{\stackrel{\circ}{V}}{\gamma} a^{3}
$$

and

$$
\begin{aligned}
b & =-\varepsilon \frac{4 \pi G}{3} \frac{\gamma}{\dot{\circ}} \frac{p_{a}}{a^{2}} \\
& =\varepsilon \gamma \frac{\dot{a} / N}{a} .
\end{aligned}
$$

Here $G$ is Newton's constant, $l_{p}=\sqrt{G \hbar}$ is the Planck length (with $c=1$ ), and $\gamma$ is the Barbero-Immirzi parameter of loop quantum gravity; its presence is purely for convenience of comparison to LQC and it can otherwise be set to $1 . \varepsilon= \pm 1$ is a factor that determines the orientation relative to a fiducial triad in terms of which $\stackrel{\circ}{q}_{a b}$ is expressed. Its presence is necessary for a consistent quantization because it ensures $-\infty<\nu<\infty$ as well as $-\infty<b<\infty$. These variables satisfy $\{b, \nu\}=2 / \hbar$. Physically, the volume of $\mathcal{V}$ is $V=2 \pi l_{p}^{2} \gamma|\nu|$, and from (16), $b$ is $\varepsilon \gamma \times$ (Hubble rate). Equivalently, since for flat FLRW the Ricci scalar is $R=-6(\dot{a} / N a)^{2}=-6(b / \gamma)^{2}$, $b$ is also a measure of spacetime curvature.

The classical dynamical trajectories given by the solution of Hamilton's equations show that $p_{\phi}$ is a constant of the motion, and

$$
\phi= \pm \kappa^{-1} \ln \left|\frac{\nu}{\nu_{0}}\right|+\phi_{0}, \quad V=V_{0} e^{ \pm \kappa\left(\phi-\phi_{0}\right)},
$$

where $\kappa=\sqrt{12 \pi G}$ and $\nu_{0}$ and $\phi_{0}$ are constants of integration. Thus the classical solutions split into a pair of disjoint expanding $(+)$ and contracting $(-)$ solutions, regarding the value of the scalar field $\phi$ as an internal emergent physical "clock". All classical solutions are therefore singular either in the "past" $(\phi \rightarrow-\infty)$ or the "future" $(\phi \rightarrow+\infty){ }^{\mathrm{h}}$

Solving the Hamiltonian constraint $H \approx 0$ gives the Friedmann equation

$$
\left(\frac{b}{\gamma}\right)^{2}=\frac{8 \pi G}{3} \rho
$$

for a flat universe, where the scalar matter energy density on the spatial slices $\Sigma$ is given by

$$
\rho=\frac{p_{\phi}^{2}}{\left.2 V\right|_{\phi} ^{2}}
$$

Here $\left.V\right|_{\phi} ^{2}$ is the volume at scalar field value $\phi$ given by (17).

\footnotetext{
g Note we have adopted the conventions used in Refs. 33, 24, which differ slightly from the normalization chosen in Ref. 22.

hActually, if one expresses (17) in terms of $\nu$, we see there are four distinct solutions, two with $\nu>0$ and two with $\nu<0$. These solutions are physically degenerate.
} 
To make the connection with loop quantum cosmology, we note that in LQC the natural phase space variables specify the symmetric connection $c$ and its conjugate triad $p$, related to the Ashtekar-Barbero $S U(2)$ connection $A_{a}^{i}$ and the densitized $\operatorname{triad} E_{i}^{a}$ by

$$
A_{a}^{i}=c \stackrel{\circ}{V}^{-1 / 3} \stackrel{\circ}{\omega}_{a}^{i}, \quad E_{i}^{a}=p \sqrt{\dot{q}} \stackrel{\circ}{V}^{-2 / 3} \stackrel{\circ}{e}_{i}^{a} .
$$

Here $\stackrel{\circ}{e}_{i}^{a}$ and $\stackrel{\circ}{\omega}_{a}^{i}$ are the fiducial triad and co-triad respectively, $\stackrel{\circ}{e}_{i}^{a} \stackrel{\circ}{\omega}_{a}^{j}=\delta_{i}^{j}$, in terms of which $\stackrel{\circ}{q}_{a b}=\dot{\circ}_{a}^{i} \dot{\omega}_{b}^{j} \delta_{i j}$. In these variables the physical volume of $\mathcal{V}$ is given by $V=|p|^{3 / 2}$, and

$$
b=\frac{c}{|p|^{1 / 2}}, \quad \nu=\varepsilon \frac{|p|^{3 / 2}}{2 \pi \gamma l_{p}^{2}} .
$$

It's easy to check that $\{c, p\}=(8 \pi G / 3) \gamma$. The variables $c$ and $p$ are related to the original geometrodynamic phase space variables by

$$
\begin{aligned}
c & =\varepsilon \frac{4 \pi G}{3} \gamma \stackrel{\circ}{V}^{-2 / 3} \frac{p_{a}}{a}, \quad p=\varepsilon \stackrel{\circ}{V}^{2 / 3} a^{2} \\
& =\varepsilon \gamma \stackrel{\circ}{V}^{1 / 3} \frac{\dot{a}}{N} .
\end{aligned}
$$

\subsection{Quantization of scalar cosmologies}

We proceed to describe two physically inequivalent ${ }^{32}$ quantizations of this classical model, a conventional, so-called "Wheeler-DeWitt" quantization, and a distinct loop quantum gravity-inspired quantization called "sLQC" (for "solvable LQC").

In order to quantize, we must choose a gauge i.e. fix the lapse $N(t)$. The classical "harmonic" gauge $N(t)=a(t)^{3}$ simplifies the Hamiltonian (14), and it is this choice that leads to the exact solvability of the loop quantization. In this gauge the Hamiltonian may be written in the variables $(b, \nu)$ as

$$
H=\frac{1}{2 V^{\circ}}\left\{-3 \pi G \hbar^{2} b^{2} \nu^{2}+p_{\phi}^{2}\right\} .
$$

This is the Hamiltonian we will quantize in the sequel.

It should be noted that in spite of the fact that $-\infty<\nu<\infty$, in both the Wheeler-DeWitt and loop quantizations the $\nu \lessgtr 0$ sectors are both disjoint and physically identical in the absence of fermions, differing only in triad orientation. Physical states may be assumed to be symmetric in $\nu$, and we will typically restrict attention to the $\nu>0$ sector. (See Ref. 32 for further discussion of this point.)

\subsubsection{Wheeler-DeWitt quantization}

The conventional quantization proceeds as usual by defining the conjugate operators

$$
\begin{array}{cc}
\hat{\nu}=\nu & \hat{b}=2 i \partial_{\nu} \\
\hat{\phi}=\phi & \hat{p}_{\phi}=-i \hbar \partial_{\phi}
\end{array}
$$


which satisfy $[\hat{b}, \hat{\nu}]=2 i$ and $\left[\hat{\phi}, \hat{p}_{\phi}\right]=i \hbar$, in accord with the Poisson brackets given above. The Hamiltonian constraint, $H \approx 0$, becomes the Wheeler-DeWitt equation $\hat{H} \Psi^{\mathrm{wdw}}=0$,

$$
\begin{aligned}
\partial_{\phi}^{2} \Psi^{\mathrm{Wdw}}(\nu, \phi) & =\kappa^{2} \frac{1}{\sqrt{|\nu|}} \nu \partial_{\nu}\left(\nu \partial_{\nu}\left(\sqrt{|\nu|} \Psi^{\mathrm{Wdw}}(\nu, \phi)\right)\right) \\
& \equiv \Theta^{\mathrm{Wdw}} \Psi^{\mathrm{Wdw}}(\nu, \phi),
\end{aligned}
$$

with a choice of operator ordering corresponding to the Laplace-Beltrami operator of the DeWitt metric on the configuration space $(\nu, \phi){ }^{\mathrm{i}}$

Group averaging leads to a solvable quantum theory in which physical states may be chosen to be "positive frequency" solutions to the quantum constraint

$$
+\hat{p}_{\phi} \Psi^{\mathrm{Wdw}}(\nu, \phi)=\hbar \sqrt{\Theta^{\mathrm{wdw}}} \Psi^{\mathrm{Wdw}}(\nu, \phi) .
$$

(The positive and negative frequency sectors are disjoint and physically equivalent.) Thus

$$
\Psi^{\mathrm{WdW}}(\nu, \phi)=U^{\mathrm{WdW}}\left(\phi-\phi_{0}\right) \Psi^{\mathrm{WdW}}\left(\nu, \phi_{0}\right)
$$

where the "propagator" in internal "time" $\phi$ is given by

$$
U^{\mathrm{WdW}}(\phi)=e^{i \sqrt{\Theta^{\mathrm{WdW}}} \phi} .
$$

The gravitational "evolution" operator $\Theta^{\mathrm{Wdw}}$ is positive and self-adjoint in the group-averaged, Schrödinger-like inner product ${ }^{j}$

$$
\left\langle\Psi^{\mathrm{WdW}} \mid \Phi^{\mathrm{WdW}}\right\rangle=\int_{-\infty}^{\infty} d \nu \Psi^{\mathrm{WdW}}(\nu, \phi)^{*} \Phi^{\mathrm{WdW}}(\nu, \phi) .
$$

Solutions may be conveniently expressed in terms of the eigenfunctions of $\Theta^{\mathrm{wdw}}$,

$$
e_{k}^{\mathrm{Wdw}}(\nu)=\frac{1}{\sqrt{4 \pi|\nu|}} e^{i k \ln \left|\frac{\nu}{\lambda}\right|}
$$

where

$$
\Theta^{\mathrm{Wdw}} e_{k}^{\mathrm{WdW}}=\omega_{k}^{2} e_{k}^{\mathrm{WdW}} .
$$

Here $\omega_{k}=\kappa|k|$, and we choose $\lambda=\sqrt{\Delta} \cdot l_{p}=\sqrt{4 \sqrt{3} \pi \gamma} \cdot l_{p} .\left(\lambda^{2}\right.$ is the so-called "area gap" of loop quantum gravity.) This specific choice for the constant $\lambda$ - necessary for dimensional reasons - plays no physical role in the Wheeler-DeWitt theory, but

${ }^{\mathrm{i}}$ This is the choice adopted in Refs. 33, 24, and is a slightly different representation than that employed in Ref. 22. In contrast to that reference, our states carry an additional factor of $\sqrt{\lambda /|\nu|}$ in order to simplify the form of the inner product.

jIt is perhaps worth some emphasis that the form of the inner product in both the WheelerDeWitt and loop quantizations depends on the chosen representation of states. For example, in some variables the inner product assumes a Klein-Gordon type form; see Refs. 34, 22 for some discussion and examples. 
is a convenient normalization for comparison with LQC. In terms of the $e_{k}^{\mathrm{Wdw}}(\nu)$, physical states may be written

$$
\begin{aligned}
\Psi^{\mathrm{Wdw}}(\nu, \phi) & =\int_{-\infty}^{\infty} d k \tilde{\Psi}^{\mathrm{Wdw}}(k) e_{k}^{\mathrm{Wdw}}(\nu) e^{i \omega_{k}\left(\phi-\phi_{0}\right)} \\
& =\Psi_{R}^{\mathrm{Wdw}}(\nu, \phi)+\Psi_{L}^{\mathrm{Wdw}}(\nu, \phi)
\end{aligned}
$$

where

$$
\begin{aligned}
& \Psi_{R}^{\mathrm{Wdw}}(\nu, \phi)=\frac{1}{\sqrt{4 \pi|\nu|}} \int_{-\infty}^{0} d k \tilde{\Psi}^{\mathrm{WdW}}(k) e^{i k\left[\ln \left|\frac{\nu}{\lambda}\right|-\kappa\left(\phi-\phi_{0}\right)\right]} \\
& \Psi_{L}^{\mathrm{WdW}}(\nu, \phi)=\frac{1}{\sqrt{4 \pi|\nu|}} \int_{0}^{\infty} d k \tilde{\Psi}^{\mathrm{Wdw}}(k) e^{i k\left[\ln \left|\frac{\nu}{\lambda}\right|+\kappa\left(\phi-\phi_{0}\right)\right]} .
\end{aligned}
$$

These "right-" and "left-"moving (in a plot of $\phi$ vs. $\nu$ ) states clearly correspond to the expanding and contracting branches of the classical solution. In the quantum theory, the $R$ and $L$ sectors are orthogonal and a-priori independent of one another. A state may be purely $R$ - (or $L$-) moving, or a superposition of the two.

For considerable further detail concerning the quantization and references to the earlier literature see Ref. 32 .

\subsubsection{Observables}

The physical ("Dirac") observables must be represented by operators that commute with the constraint $\Theta^{\mathrm{wdw}}$. The scalar momentum $\hat{p}_{\phi}$ clearly commutes with $\sqrt{\Theta^{\mathrm{wdW}}}$ via (25) and is therefore a constant of the motion as in the classical theory. The volume $\hat{\nu}$ is not. However, the corresponding "relational" observable $\left.\hat{\nu}\right|_{\phi^{*}}$ that gives the value of the volume at a fixed value $\phi^{*}$ of the scalar field is. The "Heisenbergpicture" operator $\left.\hat{\nu}\right|_{\phi^{*}}(\phi)$ that acts on states at $\phi$ is given by

$$
\left.\hat{\nu}\right|_{\phi^{*}}(\phi)=U\left(\phi^{*}-\phi\right)^{\dagger} \hat{\nu} U\left(\phi^{*}-\phi\right),
$$

where $U(\phi)$ is given by (28). Thus, for example, the physical volume of the fiducial cell $\mathcal{V}$ at $\phi^{*}$ is given by the operator $\left.\hat{\nu}\right|_{\phi^{*}}(\phi)$ whose action is

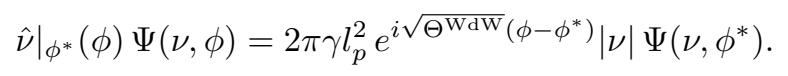

FLRW universes are, of course, classically singular - every classical solution (17) begins or ends in a zero-volume singularity with diverging energy density. In Ref. 34 it is shown that the same is true of the Wheeler-DeWitt quantum theory in the sense that the expectation value of the volume observable vanishes for generic right-moving (expanding) states as $\phi \rightarrow-\infty$, and for generic left-moving states as $\phi \rightarrow+\infty$. (This is actually evident from Eq. (33) by simply invoking the Riemann-Lebesgue lemma.) Since $\hat{p}_{\phi}$ is a constant of the motion this implies the matter energy density (19) diverges in those limits for generic states. By constrast, they showed that in sLQC the (expectation value of the) volume for generic states remains bounded away from zero, and the (expectation value of the) matter energy 
density is bounded above for generic states. ${ }^{\mathrm{k}}$ Here we will sharpen these results and demonstrate the singularity of the Wheeler-DeWitt quantization and concomitant finiteness of LQC using the consistent histories framework for quantum theory.

\subsubsection{Loop quantization}

The loop quantization proceeds by quantizing the Hamiltonian (23) on a different kinematical Hilbert space. (For details and references to the earlier literature see Ref. 32.) If $|\nu\rangle$ denotes the eigenstates of the multiplicative volume operator $\hat{\nu}$, in this inequivalent quantization one finds that $\widehat{\exp (i \lambda b)}|\nu\rangle=|\nu-2 \lambda\rangle$ acts as a translation. Quantizing the Hamiltonian constraint once again leads to

$$
\hat{p}_{\phi}^{2} \Psi(\nu, \phi)=\hbar^{2} \Theta \Psi(\nu, \phi),
$$

but now $\Theta$ is a second-order difference operator

$$
\begin{array}{r}
\Theta \Psi(\nu, \phi)=-\frac{3 \pi G}{4 \lambda^{2}}\left\{\sqrt{|\nu(\nu+4 \lambda)| \mid} \nu+2 \lambda \mid \Psi(\nu+4 \lambda, \phi)-2 \nu^{2} \Psi(\nu, \phi)\right. \\
+\sqrt{|\nu(\nu-4 \lambda)| \mid} \nu-2 \lambda \mid \Psi(\nu-4 \lambda, \phi)\} .
\end{array}
$$

Solutions to the full quantum constraint $\hat{C}=-\left[\partial_{\phi}^{2}+\Theta\right]$ therefore decompose into disjoint sets of solutions on the $\epsilon$-lattices given by $\nu=4 \lambda n+\epsilon$, where $\epsilon \in[0,4 \lambda)$. Without loss of generality we will restrict attention to the sector of the theory on the $\epsilon=0$ latice. Thus, volume in this model is discrete,

$$
\nu=4 \lambda n, \quad n \in \mathbb{Z}
$$

Group averaging leads to the physical inner product

$$
\langle\Psi \mid \Phi\rangle=\sum_{\nu=4 \lambda n} \Psi\left(\nu, \phi_{0}\right)^{*} \Phi\left(\nu, \phi_{0}\right)
$$

at some fiducial (but physically irrelevant) $\phi_{0}$. Once again the theory splits into disjoint, physically degenerate positive- and negative-frequency sectors, and as before we restrict our attention to the positive frequency sector

$$
-i \partial_{\phi} \Psi(\nu, \phi)=\sqrt{\Theta} \Psi(\nu, \phi) .
$$

Also as in the Wheeler-DeWitt theory, states in sLQC may be "propagated" by $U(\phi)=\exp (i \sqrt{\Theta} \phi)$. Relational Dirac observables in sLQC are then defined in precisely the same manner as in the Wheeler-DeWitt theory.

Similarly, quantum states may be represented in terms of the symmetric eigenfunctions $e_{k}^{(s)}(\nu)$ of $\Theta$,

$$
\Theta e_{k}^{(s)}(\nu)=\omega_{k}^{2} e_{k}^{(s)}(\nu)
$$

${ }^{\mathrm{k}}$ Prior work ${ }^{35,36}$ employing lapse $N=1$ had shown this numerically for sharply peaked states (later confirmed for generic states. ${ }^{37,38}$ ) The exact solvability of the theory in the harmonic gauge makes an analytic result possible in sLQC. 
as

$$
\Psi(\nu, \phi)=\int_{-\infty}^{\infty} d k \tilde{\Psi}(k) e_{k}^{(s)}(\nu) e^{i \omega_{k}\left(\phi-\phi_{0}\right)} .
$$

Explicit expressions for the eigenfunctions $e_{k}^{(s)}(\nu)$, based on the earlier work of Refs. 39, 40, 41, are given in 33. (See Eq. (3.14) of that reference.) Their salient properties are

(i) They are oscillatory functions of both $k$ and $\nu$, oscillating increasingly rapidly in $k$ as $\nu$ increases. They are symmetric in both $k$ and $\nu$.

(ii) At large volume (specifically, for $|\nu| \gg 2 \lambda|k|$ ), they rapidly approach a specific linear combination of corresponding Wheeler-DeWitt eigenfunctions, namely

$$
\begin{aligned}
e_{k}^{(s)}(\nu) & \simeq \sqrt{\frac{2 \lambda}{\pi|\nu|}} \cos \left(|k| \ln \left|\frac{\nu}{\lambda}\right|+\alpha(|k|)\right) \quad|\nu| \gg 2 \lambda|k| \\
& =\sqrt{2 \lambda}\left(e_{+|k|}^{\mathrm{WdW}}(\nu) e^{+i \alpha(|k|)}+e_{-|k|}^{\mathrm{WdW}}(\nu) e^{-i \alpha(|k|)}\right),
\end{aligned}
$$

where $\alpha(k)=k \ln (1-\ln k)+\frac{\pi}{4}$.

(iii) Regarded as functions of $k$, the $e_{k}^{(s)}(\nu)$ exhibit a sharp exponential ultraviolet cutoff that sets in when $2 \lambda|k| \approx|\nu|$. In other words, the eigenfunctions have support only in the wedge

$$
|k| \lesssim \frac{|\nu|}{2 \lambda}
$$

All of these features are evident in Fig. 1.

Property (ii) is worthy of note because it directly implies that all states in LQC approach a specific, symmetric linear combination of expanding and contracting Wheeler-DeWitt states

$$
\Psi(\nu, \phi) \approx \Psi_{R}^{\mathrm{WdW}}(\nu, \phi)+\Psi_{L}^{\mathrm{WdW}}(\nu, \phi) \quad\left(\begin{array}{c}
\text { large } \\
\text { volume }
\end{array}\right)
$$

at large volume. ${ }^{33}$ (See Refs. 33, 24 for considerable further detail and discussion of this point. By contrast, recall in the Wheeler-DeWitt theory the expanding and contracting branches of a generic state are completely independent of one another.) This is one signature of the quantum "bounce" characteristic of loop quantum states. Indeed, it is well known that in LQC quasiclassical states (as described in the sequel) remain peaked on a solution of the quantum-corrected Friedmann equation ${ }^{42,43}$

$$
\left(\frac{\dot{a}}{a}\right)^{2}=\frac{8 \pi G}{3} \rho\left(1-\frac{\rho}{\rho_{\max }}\right)
$$

for all values of the volume, "bouncing" from one large volume state to another rather than being swallowed by the singularity as in the classical or Wheeler-DeWitt theory; see Fig. 3. Here $\rho_{\max }$ is the maximum matter energy density defined in (47) below. Equation (45) is an indication of the remarkable fact, demonstrated in further detail in the sequel, that generic quantum states in sLQC are nonsingular ${ }^{24,32-38}$ all states "bounce", not merely quasiclassical ones. 


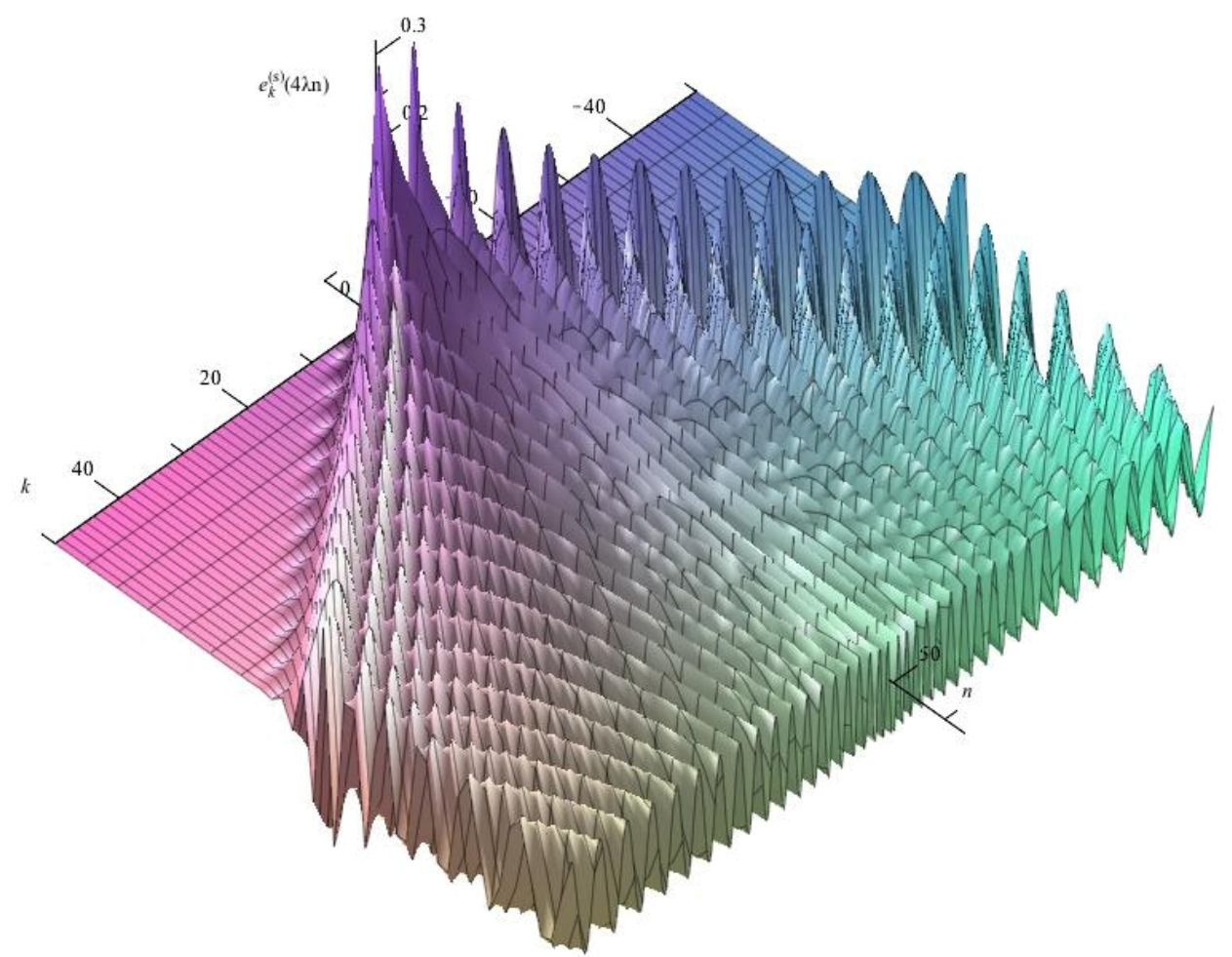

Fig. 1. Plot of the gravitational functions $e_{k}^{(s)}(\nu=4 \lambda n)$ in the $(k, n)$ plane. The volume variable $\nu=4 \lambda n$ is fundamentally discrete; the values of the eigenfunctions are plotted as continuous in both variables $k$ and $n$ for reasons of visual clarity only. The exponential ultraviolet cutoff along the lines $|k|=2|n|=|\nu / 2 \lambda|$ is clearly evident. The eigenfunctions $e_{k}^{(s)}(\nu)$ may therefore be regarded to an excellent approximation as having support only in the "wedge" $|k| \lesssim 2|n|$. It is this feature of the eigenfunctions that is ultimately responsible for the existence of a universal upper bound to the matter density.

In this regard property (iii) of the eigenfunctions is especially interesting, because it is directly responsible for the non-singular nature of sLQC. It is a manifestation of the quantum gravitational repulsion that appears at the Planck scale in LQG. In fact, one may argue heuristically that the matter energy density remains bounded in LQC by

$$
\rho_{\max }=\frac{\sqrt{3}}{32 \pi^{2} \gamma^{3}} \rho_{p}
$$

where $\rho_{p}=1 / G l_{p}^{2}$ is the Planck density. The argument proceeds as follows. The matter density is given classically by (19). It is argued in Ref. 34 that correspondingly

$$
\left\langle\left.\rho\right|_{\phi}\right\rangle=\frac{1}{2} \frac{\left\langle p_{\phi}^{2}\right\rangle}{\left\langle\left.\hat{V}\right|_{\phi} ^{2}\right\rangle}
$$


in the quantum theory. (Variations on this definition have also been discussed. ${ }^{33,34}$ ) Now, the eigenvalues of $\hat{p}_{\phi}$ are $\hbar \kappa k$, and the UV cutoff (44) on the eigenfunctions $e_{k}^{(s)}(\nu)$ requires $|k| \lesssim|\nu| / 2 \lambda$, so that with $\hat{V}=2 \pi l_{p}^{2} \gamma|\hat{\nu}|$,

$$
\begin{aligned}
\left\langle\left.\rho\right|_{\phi}\right\rangle & \sim \frac{1}{2} \frac{(\hbar \kappa|k|)^{2}}{\left(2 \pi l_{p}^{2} \gamma|\nu|\right)^{2}} \\
& \lesssim \frac{1}{2}\left(\frac{\hbar \kappa}{2 \lambda}\right)\left(\frac{|\nu|}{2 \pi \gamma l_{p}^{2}|\nu|}\right)^{2},
\end{aligned}
$$

which gives precisely the bound (47). Thus, the linear scaling of the UV cutoff on the eigenfunctions with volume leads to a universal upper bound on the expectation value (hence spectrum) of the matter energy density. It is satisfying that this simple heuristic argument based on the UV cutoff (44) reproduces precisely the value of the bound on the density found by a careful analytical argument in Ref. 34. A quite different analytical argument may be found in Ref. 33 .

\section{Consistent Histories Formulation of Canonical Quantum Cosmology}

Both the Wheeler-DeWitt quantization and sLQC are canonical quantum theories, in the sense that they describe quantum universes by states in well-defined Hilbert spaces, with physical observables represented (Dirac) operators on those Hilbert spaces. This means that each may be given a consistent histories formulation that closely resembles that of non-relativistic quantum theory.

Indeed, the presence in these models of a variable that behaves as an emergent internal matter "clock", the scalar field $\phi$ (via $|\Psi(\phi)\rangle=U(\phi)|\Psi\rangle)$, only encourages that association. We emphasize, however, that that association is not necessary for the formulation of the consistent histories framework. What matters is that well-defined quantum states and observable operators are present which permit the definition of class operators and branch wave functions out of which a decoherence functional may be constructed. The decoherence functional can then be employed to make predictions concerning patterns of correlation between any of the observable quantities, whether or not they are necessarily ordered in some "time" variable. The existence of an internal time, however, does supply a convenient context for the physical interpretation of the resulting theory.

\subsection{Decoherence functional}

In order to define the decoherence functional for these theories we must first construct class operators and branch wave functions for quantum histories defined by histories of values of the theory's Dirac observables. The constructions are identical in each theory so long as they are expressed in terms of the gravitational constraint operator $\Theta$ and its eigenfunctions. The differing physical predictions then arise because of the sharply distinct behavior of these objects in the two theories. 
We therefore proceed to formulate the decoherence functional in terms of $\Theta$ and its eigenfunctions alone, and apply the construction to contrast the physical predictions of the two theories in the sequel.

In this simple FLRW model our operators are $\left\{\hat{\phi}, \hat{p}_{\phi}, \hat{b}(\right.$ or $\left.\widehat{\exp (i \lambda b)}), \hat{\nu}\right\}$ with corresponding relational Dirac observables given by the equivalents of (34). Given its relevance to the question of the singularity of the universe we will concentrate most of our attention on $\hat{\nu}$, but parallel constructions are available for any observable.

The definition of class operators is based on the spectral decomposition of observables. In the case of the volume operator

$$
\begin{aligned}
\hat{\nu}^{\mathrm{WdW}} & =\int_{-\infty}^{\infty} d \nu^{\prime} \nu^{\prime}\left|\nu^{\prime}\right\rangle\left\langle\nu^{\prime}\right| \\
& =\int_{-\infty}^{\infty} d \nu^{\prime} \nu^{\prime} P_{\nu^{\prime}}^{\nu},
\end{aligned}
$$

with

$$
\begin{aligned}
\left\langle\nu \mid \nu^{\prime}\right\rangle & =\delta\left(\nu-\nu^{\prime}\right) \\
e_{k}^{\mathrm{Wdw}}(\nu) & =\left\langle\nu \mid k^{\mathrm{wdw}}\right\rangle
\end{aligned}
$$

and $P_{\nu^{\prime}}^{\nu}$ a volume projection on the eigenket $\left|\nu^{\prime}\right\rangle$. (We work with a normalization appropriate to the full range $-\infty<\nu<\infty$ and take all states to be symmetric in $\nu$.$) By contrast, in sLQC volume is discrete:$

$$
\begin{aligned}
\hat{\nu} & =\sum_{\nu=4 \lambda n} \nu|\nu\rangle\langle\nu| \\
& =\sum_{\nu^{\prime}=4 \lambda n^{\prime}} \nu^{\prime} P_{\nu^{\prime}}^{\nu} .
\end{aligned}
$$

and

$$
\begin{aligned}
\left\langle\nu \mid \nu^{\prime}\right\rangle & =\delta_{n, n^{\prime}} \\
e_{k}^{(s)}(\nu) & =\left\langle\nu \mid k^{(s)}\right\rangle .
\end{aligned}
$$

In the sequel $\hat{\nu}$ will refer to whichever theory is appropriate in context, with integrals in the Wheeler-DeWitt theory and sums in sLQC. For example, the projection onto a range of values $\Delta \nu$ will be one of

$$
P_{\Delta \nu}^{\nu}= \begin{cases}\int_{\nu \in \Delta \nu} d \nu|\nu\rangle\langle\nu| & \text { Wheeler-DeWitt } \\ \sum_{\nu \in \Delta \nu}|\nu\rangle\langle\nu| & \text { slQC }\end{cases}
$$

"Heisenberg" projections may be defined via the propagator $U(\phi)=\exp (i \sqrt{\Theta} \phi)$ as

$$
P_{\Delta a_{k}^{\alpha}}^{\alpha}(\phi)=U\left(\phi-\phi_{0}\right)^{\dagger} P_{\Delta a_{k}^{\alpha}}^{\alpha} U\left(\phi-\phi_{0}\right),
$$

where $\phi_{0}$ is a fiducial (but physically irrelevant) value of the scalar field at which the quantum state is defined. Class operators may then be defined as in Eqs. (2-3). 
For example, the class operator for the relational history in which the volume is in $\Delta \nu$ when the scalar field has value $\phi^{*}$ is

$$
C_{\left.\Delta \nu\right|_{\phi^{*}}}=U\left(\phi^{*}-\phi_{0}\right)^{\dagger} P_{\Delta \nu}^{\nu} U\left(\phi^{*}-\phi_{0}\right) .
$$

It turns out such class operators offer an illuminating perspective on relational observables such as $\left.\nu\right|_{\phi^{*}}(\phi)$, a point to which we shall return later. Similarly, the class operator describing a coarse-grained trajectory $\nu(\phi)$, for which the volume is in $\Delta \nu_{1}$ at scalar field value $\phi_{1}, \Delta \nu_{2}$ at $\phi_{2}$, and so in, is

$$
C_{\left.\Delta \nu_{1}\right|_{\phi_{1}} ;\left.\Delta \nu_{2}\right|_{\phi_{2}} ; \cdots ;\left.\Delta \nu_{n}\right|_{\phi_{n}}}=P_{\Delta \nu_{1}}^{\nu}\left(\phi_{1}\right) P_{\Delta \nu_{2}}^{\nu}\left(\phi_{2}\right) \cdots P_{\Delta \nu_{n}}^{\nu}\left(\phi_{n}\right) \text {. }
$$

Branch wave functions corresponding to a history $h$ are then defined as in (7) by

$$
\left|\Psi_{h}(\phi)\right\rangle=U\left(\phi-\phi_{0}\right) C_{h}^{\dagger}|\Psi\rangle
$$

which is everywhere a solution of the theory's Wheeler-DeWitt equation (i.e. annihilated by the quantum constraint.) The decoherence functional in the case of a pure "initial" state defined on a minisuperspace surface of constant $\phi^{1}$ may then be defined simply as

$$
d\left(h, h^{\prime}\right)=\left\langle\Psi_{h^{\prime}} \mid \Psi_{h}\right\rangle,
$$

using the group-averaged inner product on states. Consistent or decoherent families of histories then satisfy (1) on all pairs of histories $h$ in some exclusive, exhaustive family $\{h\}$. As discussed above, the decoherence functional provides an objective, observer-independent measure of the interference among histories in the family. When that interference vanishes, each history in the family may be assigned the probability $p(h)=d(h, h)$. Otherwise, quantum mechanics says that the physical question posed by the family of histories simply has no sensible answer within quantum theory.

Let us see how this is accomplished in a series of examples in keeping with the plan laid out in section 2.1.

\subsection{Consistent histories in quantum cosmology: other models}

sLQC has also been used as the basis for a spin-foam-like "path integral" formulation of loop quantum cosmology. ${ }^{32,39-41}$ (See also related work. ${ }^{44}$ ) As remarked at the end of section 2, a consistent histories formulation may also be given for theories defined via path-integrals, as has been done (for example) for Bianchi IX cosmologies and other other models. ${ }^{5,7-11,13,45}$ These constructions serve as the template for a consistent histories formulation of spin foam loop quantum cosmology ${ }^{25,46,47}$ and of spin foam loop quantum gravity (as in Ref. 31, which is directly modeled on Refs. $5,6,13$.) We do not, however, have the space to describe these path-integral consistent histories theories here.

\footnotetext{
${ }^{1}$ See 22 for brief discussion of this point.
} 
It should also be mentioned that the physical predictions of the consistent histories formulation have been compared to a de Broglie-Bohm quantization of FLRW that closely parallels the work reviewed herein. ${ }^{48,49}$ See, however, Ref. 24 for some pertinent brief discussion.

\section{Applications}

\subsection{Probabilities, histories, and relational observables}

Relational ("Dirac") observables are the physical quantities about which theories with constraints make predictions, and (by definition) must commute with those constraints. It might have been thought that the class operators for volume given in (56-57) should have been constructed directly from the Dirac observable (34). While that indeed is an option, an alternative point of view is that histories provide a natural framework within which to understand the emergence of such relational observables in theories with an emergent "time" evolution. ${ }^{22}$

To see how they arise naturally in a histories framework, consider a self-adjoint operator $\hat{A}$ that does not commute with the constraint - for example, $\hat{\nu}$, in the models we have been discussing - with spectral decomposition (assuming for definiteness that $\hat{A}$ has a purely discrete spectrum)

$$
\hat{A}=\sum_{a} a P_{a}
$$

where $P_{a}=|a\rangle\langle a|$. The class operator corresponding to histories in which $\hat{A}$ has values in one of the complete set of disjoint of intervals of eigenvalues $\{\Delta a\}$ at $\phi=\phi^{*}$ are

$$
C_{\left.\Delta a\right|_{\phi^{*}}}=U\left(\phi^{*}-\phi_{0}\right)^{\dagger} P_{\Delta a}^{A} U\left(\phi^{*}-\phi_{0}\right),
$$

with the corresponding branch wave functions defined as in (58). Because the class operators are simply one "time" $(\phi)$ projections, the branch wave functions always decohere - the family is consistent:

$$
\begin{aligned}
d\left(\Delta a, \Delta a^{\prime}\right) & =\left\langle\Psi \Psi_{\left.\Delta a^{\prime}\right|_{\phi^{*}}} \mid \Psi_{\left.\Delta a\right|_{\phi^{*}}}\right\rangle \\
& =\left\langle\Psi\left|C_{\left.\Delta a^{\prime}\right|_{\phi^{*}}} C_{\left.\Delta a\right|_{\phi^{*}}}^{\dagger}\right| \Psi\right\rangle \\
& =\left\langle\Psi\left|U\left(\phi^{*}-\phi_{0}\right)^{\dagger} P_{\Delta a^{\prime}}^{A} U\left(\phi^{*}-\phi_{0}\right) U\left(\phi^{*}-\phi_{0}\right)^{\dagger} P_{\Delta a}^{A} U\left(\phi^{*}-\phi_{0}\right)\right| \Psi\right\rangle \\
& =\left\langle\Psi\left(\phi^{*}\right)\left|P_{\Delta a^{\prime}}^{A} P_{\Delta a}^{A}\right| \Psi\left(\phi^{*}\right)\right\rangle \\
& =\left\langle\Psi\left(\phi^{*}\right)\left|P_{\Delta a}^{A}\right| \Psi\left(\phi^{*}\right)\right\rangle \delta_{\Delta a^{\prime}, \Delta a} \\
& =p_{\Delta a}\left(\phi^{*}\right) \delta_{\Delta a^{\prime}, \Delta a}
\end{aligned}
$$

where $p_{\Delta a}\left(\phi^{*}\right)$ is the probability that $a \in \Delta a$ when $\phi=\phi^{*}$. For example, since we have assumed $\hat{A}$ has a discrete spectrum if we set $\Delta a=\{a\}$, a single eigenvalue, then

$$
p_{a}\left(\phi^{*}\right)=\left|\left\langle a \mid \Psi\left(\phi^{*}\right)\right\rangle\right|^{2}
$$


as one might expect, ${ }^{\mathrm{m}}$ with a similarly expected expression if $\hat{A}$ has a continuous spectrum; see Ref. 22 for details in that case.

To see the connection with relational observables, let us calculate the average value of $\hat{A}$ at $\phi^{*}$ :

$$
\begin{aligned}
\left.\langle\hat{A}\rangle\right|_{\phi^{*}} & =\sum a p_{a}\left(\phi^{*}\right) \\
& =\left\langle\Psi\left(\phi^{*}\right)\left|\sum_{a} a P_{a}^{A}\right| \Psi\left(\phi^{*}\right)\right\rangle \\
& =\left\langle\Psi\left|U\left(\phi^{*}-\phi_{0}\right)^{\dagger} \hat{A} U\left(\phi^{*}-\phi_{0}\right)\right| \Psi\right\rangle \\
& =\left\langle\Psi|\hat{A}|_{\phi^{*}} \mid \Psi\right\rangle,
\end{aligned}
$$

the expectation value of the relational observable $\left.\hat{A}\right|_{\phi^{*}}$ corresponding to $\hat{A}$ in the state $|\Psi\rangle$. Probabilities for histories of values of $\hat{A}$ (which does not commute with the constraint) are naturally expressed in terms of the corresponding relational observable $\left.\hat{A}\right|_{\phi^{*}}$ (which does). Had we not known about Dirac observables the histories formulation would have led us to consider them.

\subsection{Scalar momentum}

The scalar field momentum $p_{\phi}$ is a constant of the motion in the classical theory, where $\left\{p_{\phi}, H\right\}=0$, and similarly in the quantum theory, $\left[\hat{p}_{\phi}, \Theta\right]=0$. How does this constancy manifest in the consistent histories formulation?

If we are only interested in the distribution of probabilities for values of $\hat{p}_{\phi}$ at a single $\phi=\phi^{*}$, we might construct the relational observable $\left.\hat{p}_{\phi}\right|_{\phi^{*}}$. However, because $\hat{p}_{\phi}$ commutes with the constraint, $\left.\hat{p}_{\phi}\right|_{\phi^{*}}=\hat{p}_{\phi}$, and correspondingly the probability $p_{\Delta p_{\phi}}\left(\phi^{*}\right)$ for $\hat{p}_{\phi}$ to have values in $\Delta p_{\phi}$ at $\phi^{*}$ is independent of $\phi^{*}$, in keeping with $p_{\phi}$ being a constant of the motion.

Similarly, to the question "what is the likelihood $p_{\phi}$ is in $\Delta p_{\phi ; 1}$ at $\phi_{1}, \Delta p_{\phi ; 2}$ at $\phi_{2}, \ldots$ (etc.)" corresponds the class operator

$$
C_{\left.\Delta p_{\phi ; 1}\right|_{\phi_{1}} ; \Delta p_{\phi ; 2}||_{2} ; \cdots ;\left.\Delta p_{\phi ; n}\right|_{\phi_{n}}}=P_{\Delta p_{\phi ; 1}}^{p_{\phi}}\left(\phi_{1}\right) P_{\Delta p_{\phi ; 2}}^{p_{\phi}}\left(\phi_{2}\right) \cdots P_{\Delta p_{\phi ; n}}^{p_{\phi}}\left(\phi_{n}\right),
$$

Since $P_{\Delta p_{\phi}}^{p_{\phi}}(\phi)=P_{\Delta p_{\phi}}^{p_{\phi}}$, all such class operators are zero unless all of the intervals $\Delta p_{\phi ; i}$ are equal, in which case each such class operator is the simple projection $P_{\Delta p_{\phi ;}}^{p_{\phi}}$. The family of all such class operators clearly decoheres for any initial state, the corresponding probabilities $p_{\Delta p_{\phi ; i}}$ all constants (i.e. independent of $\phi$ ). This is the meaning of the statement that $p_{\phi}$ is a constant of the motion in the quantum theory. (Constants of motion in generalized consistent histories quantum theory are discussed further in Ref. 12.)

\footnotetext{
${ }^{m}$ Precisely because of this expectation, it is crucial to emphasize that the simple form of this result, which we have derived from the decoherence functional, is directly connected with the simple form of the Schrödinger-like form of the inner product in this representation. As noted above, in other representations the inner product can take on e.g. a Klein-Gordon type form, and therefore the formula for the probability does as well. This illustrates the importance of placing quantum prediction in a coherent, self-consistent frame. ${ }^{13,50}$
} 


\subsection{Volume at a single value of $\phi$}

Classically, one may express the volume $\nu$ of the fiducial cell as a function $\nu(\phi)$ as in (17). It might seem natural then to ask the quantum question "what is the probability the volume $\nu$ is in the interval $\Delta \nu$ at scalar field value $\phi^{*}$ ?" The class operator corresponding to this question was given in (56). According to the calculation of (63) these single- $\phi$ histories decohere with corresponding probabilities

$$
p_{\Delta \nu}\left(\phi^{*}\right)=\left\langle\Psi_{\left.\Delta \nu\right|_{\phi^{*}}} \mid \Psi_{\left.\Delta \nu\right|_{\phi^{*}}}\right\rangle .
$$

In the Wheeler-DeWitt case this is, explicitly,

$$
p_{\Delta \nu}^{\mathrm{WdW}}\left(\phi^{*}\right)=\int_{\Delta \nu} d \nu\left|\psi^{\mathrm{Wdw}}\left(\nu, \phi^{*}\right)\right|^{2} .
$$

This probability is calculated explicitly for semi-classical Wheeler-DeWitt states (i.e. states peaked on a particular classical trajectory) in Ref. 22. In the case of a superposition of right- (expanding) and left- (contracting) moving states (as in (73)), an example of the result is shown in Figure 2. ${ }^{21,22}$ In loop quantum cosmology the equivalent expression is

$$
p_{\Delta \nu}^{\mathrm{LQC}}\left(\phi^{*}\right)=\sum_{\nu \in \Delta \nu}\left|\psi\left(\nu, \phi^{*}\right)\right|^{2} .
$$

This is again calculated for the case of "quasiclassical" loop quantum states that approach a symmetric superposition of semiclassical Wheeler-DeWitt states at large volume in Ref. 24. (For discussion of the usage of the term "quasiclassical" in this context see Sec. 5.5.)

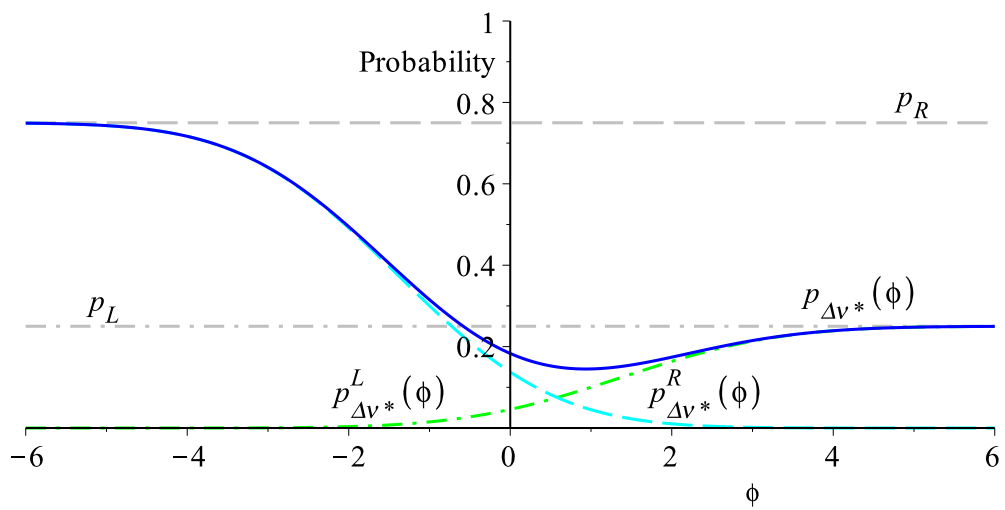

Fig. 2. The behavior of $p_{\Delta \nu^{*}}(\phi)$, the probability that the quantum universe will be found in the interval $\Delta \nu^{*}=\left[0, \nu^{*}\right]$ (i.e. at small volume) for a sample superposition of expanding $(R)$ and contracting $(L)$ semiclassical states both peaked at large volume near $\phi=0 . p_{L}$ and $p_{R}$ give the relative "amount" of each component in the superposition, so that $p_{L}+p_{R}=1$ (cf. Eq. (73).) This plot may appear to imply the possibility of a "quantum bounce", since at any given $\phi$ there is a non-zero probability that the universe may be found with volume $\nu>\nu^{*}$. A more careful consistent histories analysis shows that this possibility is not realized: the probability that the universe has large volume in both the "past" and "future" is zero. ${ }^{21,22}$ 


\subsection{Cosmological trajectories}

The class operator specifying the volume of the fiducial cell at a sequence of values of $\phi$ given in (57) describes a (coarse-grained) cosmological trajectory $\nu(\phi)$. (See Fig. 3a for some examples.) Because such class operators are not simply projections, the corresponding branch wave functions will not in general decohere, and in these simple models probabilities can not in general be assigned to the family of trajectories they describe, as is typical in quantum theory. Nonetheless, there are several physically important examples for which they do decohere.

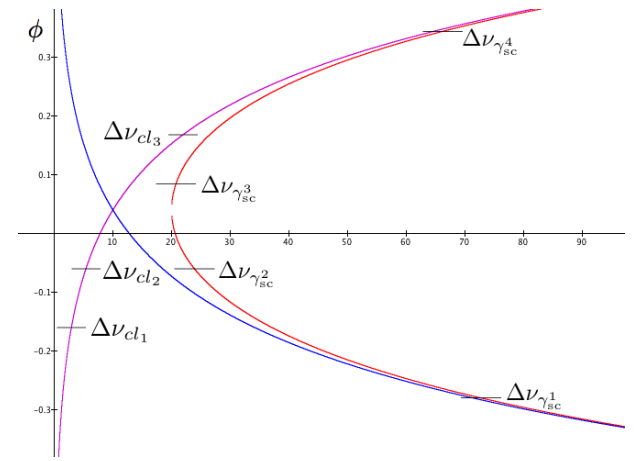

(a) Coarse-grained trajectories

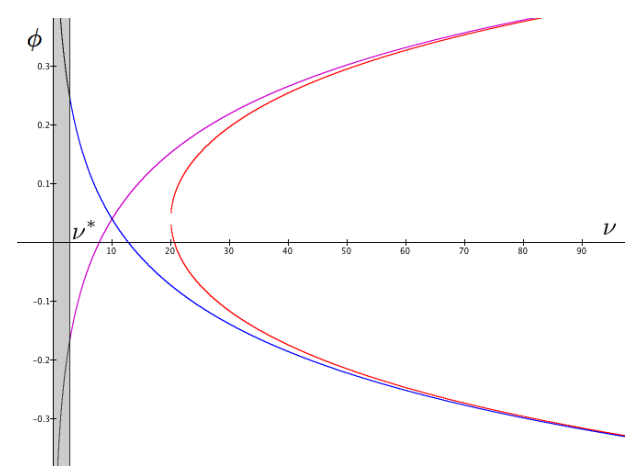

(b) Coarse-graining by singularity

Fig. 3. Coarse-grainings of the FLRW minisuperspace. The left-hand plot shows an expanding (R-moving) and collapsing (L-moving) classical trajectory as well as the corresponding "classical" loop quantum trajectory i.e. solution to the effective Friedmann equation (46). Also depicted are coarse-grainings by ranges of values of the volume at different values of the scalar field for two histories. The first is a coarse-grained history $\left(\Delta \nu_{c l_{1}}, \Delta \nu_{c l_{2}}, \Delta \nu_{c l_{3}}\right)$ describing a quantum universe peaked on an expanding classical trajectory. The second history $\left(\Delta \nu_{\gamma_{\mathrm{sc}}^{1}}, \Delta \nu_{\gamma_{\mathrm{sc}}^{2}}, \ldots\right)$ describes a loop quantum trajectory characterized by a bounce, which is peaked on symmetrically related expanding and collapsing classical trajectories at large $|\phi|$. The right-hand plot shows the same trajectories as well as a coarse-graining suitable for studying the probability that the universe assumes large or small volume. The volume $\nu$ is partitioned into the range $\Delta \nu^{*}=\left[0, \nu^{*}\right]$ (the shaded region in the figure) and its complement $\overline{\Delta \nu^{*}}=(0, \infty)$. The quantum universe may be said to attain small volume if the probability for the branch wave function $\left|\Psi_{\Delta \nu^{*}}(\phi)\right\rangle$ is near unity while that for $\left|\Psi \overline{\Delta \nu^{*}}(\phi)\right\rangle$ is near zero for arbitrary choices of $\nu^{*}$. Conversely, the universe may be said to attain arbitrarily large volume over some range of $\phi$ if the probability for $\left|\Psi \overline{\Delta \nu^{*}}(\phi)\right\rangle$ is near unity for arbitrary choice of $\nu^{*}$ over that range of $\phi$.

The simplest way in which decoherence of cosmological trajectories obtains is if the quantum state remains peaked along a particular individual trajectory, here imagined as a classical trajectory for convenience - where "classical" means a bouncing solution of the effective Friedmann equation (46) in the case of sLQC, which approaches the corresponding general relativistic solutions at large volume. (See the remarks in Section 5.5 immediately below.) Consider a coarse-graining on a set of slices $\left\{\phi_{1}, \phi_{2}, \ldots, \phi_{n}\right\}$ by a set of intervals in volume $\left\{\Delta \nu_{i_{k}}, k=1 \ldots n\right\}$ chosen in 
such a way that on each slice $\phi_{k}$ one of the ranges $\Delta \nu_{c l_{k}}$ straddles the trajectory. If $\Delta \nu_{c l_{k}}$ is wider than the width of the quantum state at $\phi_{k}$, then essentially the only non-zero branch wave function is

$$
\left|\Psi_{c l}\right\rangle=P_{\Delta \nu_{c l_{n}}}^{\nu}\left(\phi_{n}\right) \cdots P_{\Delta \nu_{c l_{2}}}^{\nu}\left(\phi_{2}\right) P_{\Delta \nu_{c l_{1}}}^{\nu}\left(\phi_{1}\right)|\Psi\rangle
$$

The family of histories (classical,non-classical) therefore decoheres and quasiclassical behavior for such a state is predicted with probability one. Attempts to specify the trajectory too finely (relative to the width of the quantum state) destroys the decoherence. This is important: quantum theory has no predictions for the trajectory followed even for quasiclassical states if it is too precisely specified. (This is merely the uncertainty principle manifesting itself in the context of quantum cosmology.)

A more detailed discussion of what it means for a state to "follow a trajectory" in generalized quantum theory is given in Ref. 24. A similar but more sophisticated analysis may be given for WKB states; see Refs. 6, 13 for details.

In this review we will describe several important examples of scenarios involving quantum histories of volume evolving with the scalar field $\phi$.

\subsection{Quasiclassical trajectories}

We intend by "quasiclassical state" to mean a quantum state that is peaked on some classical trajectory (17) at large volume. (An explicit example of such a state is given in Eq. (3.37) of Ref. 22.)

We characterize quasiclassical states in this way for the following reason. Recall that in the Wheeler-DeWitt quantization, generic quantum states (32) are a superposition of orthogonal and independent right- and left-moving (expanding and contracting) branches. In loop quantum cosmology, on the other hand, all quantum states approach a specific symmetric superposition of right- and left-moving Wheeler-DeWitt states at large volume, corresponding to the quantum "bounce" of loop quantum states.

A "quasiclassical" state in the Wheeler-DeWitt quantization is one which is peaked on either an expanding or contracting classical solution at large volume for all values of $\phi$. In the generic case that the state is a superposition of right- and left-moving branches, such a state will be peaked on a contracting classical solution as $\phi \rightarrow-\infty$, and an expanding solution as $\phi \rightarrow \infty$. If the state is purely right- or left-moving, then only one of these cases will hold.

By contrast, since loop quantum states always approach a superposition of rightand left-moving branches, "quasiclassical" states in LQC will be peaked on a solution of the "effective" Friedmann equation (46), which approaches a classical contracting solution at large volume as $\phi \rightarrow \infty$ and an expanding solution as $\phi \rightarrow+\infty$, as in the generic Wheeler-DeWitt case. By the analysis of states which remain peaked on a trajectory given above, then, a sufficiently coarse-grained family of trajectory histories will decohere for such "quasiclassical" states, predicting quasiclassical behavior at large volume with unit probability, so long as the trajectory is not too finely specified. (See Refs. 22, 24 for a more in-depth discussion.) 
(Not that this usage therefore does not imply that a "quasiclassical" state necessarily follows a classical (i.e. general relativistic) trajectory throughout its evolution. In the Wheeler-DeWitt quantum theory, such states will in fact track classical solutions all the way to the singularity, while in the case of SLQC, states which track classical general relativistic trajectories at large volume are connected by the "bounce" in the deep quantum regime along a solution to the effective Friedmann equation.)

\subsection{Volume singularity}

In order to assess whether or not a given quantum cosmological model is singular a specific criterion for singularity must be given. In these simple homogeneous isotropic models there are relatively few available, and they are clearly connected: does the volume $\nu$ of the fiducial cell become 0 ? Does the curvature $b$ diverge? Does the matter density $\rho$ (given classically by (19)) diverge? The latter definition of "quantum singularity" seems the most physical, but it does require adopting a specific definition for the matter energy density operator. Several choices are considered in Ref. 34. For each of these choices essentially the same underlying physics comes into play: as $p_{\phi}$ is a constant of the motion in both the classical and the quantum theory, and the density $\left.\rho\right|_{\phi}$ is essentially a ratio of (the square of) $p_{\phi}$ to (the square of) volume, $\left.\rho\right|_{\phi}$ will remain bounded above if the volume remains bounded below, and will diverge if the volume becomes zero. These arguments are made precise in Refs. 35, 36, 34, where it is shown that the expectation value of the volume inevitably becomes 0 for generic states in the Wheeler-DeWitt theory, and therefore the expectation value of the matter energy density diverges. By contrast, in sLQC the expectation value of the density is bounded above by the critical value (47) given by the "moral" argument of Ref. 33 and Eq. (49) due to the linear scaling of the ultraviolet cutoff on the constraint eigenfunctions; see Refs. 35, 36, 34, 32, and Ref. 33 for a different proof.

In Refs. 22, 24 the question of the singularity of the universe is addressed in the consistent histories framework, in which quantum questions concerning multiple values of $\phi$-i.e. concerning the singularity of quantum histories of the universe - may be framed and confidently answered. The most direct course would be to consider ranges of eigenvalues of the density operator and show that histories for which the density diverges have probability zero (or unity, in the Wheeler-DeWitt theory.) However, this course is not available as the spectrum of the density operator is not currently known in either theory.

Fortunately, as discussed above, at least in these models the behavior of the matter density is directly tied to the behavior of the volume of the fiducial cell, and so the analysis of the singularity of these quantum universes can instead be given in terms of the volume. ${ }^{\mathrm{n}}$ We will show that in the Wheeler-DeWitt quantum theory

$\overline{{ }^{\mathrm{n}} \text { Alternately, an analysis analagous to }}$ what we give here can be perfomed for the curvature $b$ 
all states are inevitably singular, while in the loop quantization, generic quantum states - quasiclassical or not - "bounce" from large volume to large volume. This, of course, was already known. However, the consistent histories analysis brings new, potentially sharper tools to the problem, and sheds additional light on the theory, as will be discussed in the conclusion.

The analysis proceeds as follows. The question of whether the universe becomes singular is, in these models, equivalent to the question of whether the volume becomes 0 . Therefore, partition $|\nu|$ (recall all quantum states are symmetric in $\nu$ ) into complementary, disjoint ranges $\left\{\Delta \nu^{*}, \overline{\Delta \nu^{*}}\right\}$, where $\Delta \nu^{*}=\left[0, \nu^{*}\right]$ and its complement $\overline{\Delta \nu^{*}}=\left(\nu^{*}, \infty\right)$, where $\nu^{*}$ is any arbitrary volume. (See Fig. 3b.) The universe has small volume at some scalar field value $\phi^{*}$ if $|\nu| \in \Delta \nu^{*}$ at $\phi^{*}$, and large volume if $|\nu| \in \overline{\Delta \nu^{*}}$. The corresponding branch wave functions are

$$
\left|\Psi_{\left.\Delta \nu^{*}\right|_{\phi^{*}}}(\phi)\right\rangle=U\left(\phi-\phi^{*}\right) P_{\Delta \nu^{*}}^{\nu}\left|\Psi\left(\phi^{*}\right)\right\rangle
$$

and its complement; the probability the volume is in $\Delta \nu^{*}$ given the state $|\Psi\rangle$ is then

$$
\begin{aligned}
p_{\Delta \nu^{*}}(\phi) & =\left\langle\Psi_{\left.\Delta \nu^{*}\right|_{\phi^{*}}}(\phi) \mid \Psi_{\left.\Delta \nu^{*}\right|_{\phi^{*}}}(\phi)\right\rangle \\
& = \begin{cases}\int_{0}^{\nu^{*}} d \nu\left|\psi^{\mathrm{WdW}}(\nu, \phi)\right|^{2} & \text { Wheeler-DeWitt } \\
\sum_{|\nu| \in \Delta \nu^{*}}|\psi(\nu, \phi)|^{2} & \text { sLQC },\end{cases}
\end{aligned}
$$

with $p_{\Delta \nu^{*}}(\phi)=1-p_{\overline{\Delta \nu^{*}}}(\phi)$. These probabilities are evaluated explicitly for quasiclassical Wheeler-DeWitt and loop quantum states in Refs. 22, 24. We are interested here, however, in the question of the singularity of such states.

We begin with the Wheeler-DeWitt theory. It is shown in detail in Ref. 22 that for purely left- or right-moving states

$$
\begin{array}{ll}
\lim _{\phi \rightarrow-\infty} p_{\Delta \nu^{*}}^{L}(\phi)=0 & \lim _{\phi \rightarrow+\infty} p_{\Delta \nu^{*}}^{L}(\phi)=1 \\
\lim _{\phi \rightarrow-\infty} p_{\Delta \nu^{*}}^{R}(\phi)=1 & \lim _{\phi \rightarrow+\infty} p_{\Delta \nu^{*}}^{R}(\phi)=0
\end{array}
$$

for any fixed $\nu^{*}$. This result may be understood most easily by examining Eqs. (33). From the Riemann-Lebesgue lemma it is clear that all right-/left-moving states are "sucked in" to the singularity along the classical trajectories (17). This means, perhaps unsurprisingly, that generic expanding states are inevitably singular as $\phi \rightarrow-\infty$, and contracting states are inevitably singular as $\phi \rightarrow+\infty$. Two points merit emphasis. First, while the result is expected, it has been here derived within a well-defined framework for quantum prediction. Second, there is the question of the role of the limits $|\phi| \rightarrow \infty$. It may indeed be the case that some quantum states become singular at finite $\phi$. The limit $|\phi| \rightarrow \infty$ serves to show that the singularity is a generic prediction for all states in the theory.

conjugate to the volume. 
It is noteworthy, however, that generic states in the Wheeler-DeWitt theory are actually superpositions of expanding and contracting states. What are the corresponding probabilities then? Indeed, if one writes

$$
|\Psi\rangle=\sqrt{p_{L}}\left|\Psi_{L}\right\rangle+\sqrt{p_{R}}\left|\Psi_{R}\right\rangle
$$

one finds

$$
p_{\Delta \nu^{*}}(\phi)=p_{\Delta \nu^{*}}^{L}(\phi)+p_{\Delta \nu^{*}}^{R}(\phi),
$$

with $p_{\Delta \nu}^{L, R}(\phi)$ given as above, and

$$
\lim _{\phi \rightarrow-\infty} p_{\Delta \nu^{*}}(\phi)=p_{R} \quad \text { and } \quad \lim _{\phi \rightarrow+\infty} p_{\Delta \nu^{*}}(\phi)=p_{L} .
$$

See Fig. 2 for an example (and Ref. 22 for an explicit formula for quasiclassical states.)

By contrast, in loop quantum cosmology one finds ${ }^{24}$ instead that, for generic states

$$
\begin{array}{ll}
\lim _{\phi \rightarrow-\infty} p_{\Delta \nu^{*}}(\phi)=0 & \lim _{\phi \rightarrow+\infty} p_{\Delta \nu^{*}}(\phi)=0 \\
\lim _{\phi \rightarrow-\infty} p_{\overline{\Delta \nu^{*}}}(\phi)=1 & \lim _{\phi \rightarrow+\infty} p_{\overline{\Delta \nu^{*}}}(\phi)=1 .
\end{array}
$$

from which it is clear that loop quantum states invariably bounce. The argument is once again based on the behavior of the eigenfunctions $e_{k}^{(s)}(\nu)$ inserted into (42). The UV cutoff in the eigenfunctions ensures that for any finite volume $\nu^{*}$, all states in sLQC vanish as $|\phi| \rightarrow \infty$, again on account of Riemann-Lebesgue. In addition, essentially because the eigenfunctions vanish at $\nu=0$, the probability the volume of the fiducial cell is precisely 0 is 0 for all $\phi: p_{\nu=0}(\phi)=0$. These universes never become singular for any value of $\phi$.

\subsection{Quantum bounce}

We have shown how, within a consistent histories framework, it may be demonstrated that the probability that the universe achieves zero volume is zero, while the probability that generic quantum states achieve arbitrarily large volume in both limits $|\phi| \rightarrow \infty$ is unity: all quantum states in sLQC "bounce" from large volume to large volume.

By contrast, in the Wheeler DeWitt quantum theory, we showed that all rightmoving (expanding) states inevitably assume zero volume as $\phi \rightarrow-\infty$, and contracting states do so as $\phi \rightarrow+\infty$, and are therefore singular. However, a superposition of expanding and contracting states leads to a probability for the universe having small volume that is in general never unity, just as it is never zero. This is suggestive that, in the Wheeler-DeWitt quantum theory, such a superposition state leads to a non-zero probability that it, too, might "bounce" from large volume to large volume (with probability $p_{\text {bounce }}=p_{L} \cdot p_{R}$ ).

This possibility is not realized, however - all quantum states in the WheelerDeWitt quantum theory are singular, just as all states in sLQC are non-singular. To 
understand why this is so it is important to recognize that the question of whether or not a quantum universe bounces is not a question about a single value of $\phi-$ it is a question about a coarse-grained trajectory: does the universe have a large volume at (at least) two values of $\phi$, one in the "past" and one in the "future"? This is a genuinely quantum question, and only has a definite quantum answer in the instance that the corresponding family of histories decoheres. ${ }^{20,22,24}$ We shall show, in fact, that in an appropriate limit it does, and that indeed loop quantum universes are generically non-singular and Wheeler-DeWitt universes are singular.

To pose the question concretely, consider partitions of the volume $\left\{\Delta \nu_{1}^{*}, \overline{\Delta \nu_{1}^{*}}\right\}$ and $\left\{\Delta \nu_{2}^{*}, \overline{\Delta \nu_{2}^{*}}\right\}$ on two minisuperspace $\phi$-slices $\phi_{1}$ and $\phi_{2}$. The class operator describing a "bounce" is then

$$
C_{\text {bounce }}\left(\phi_{1}, \phi_{2}\right)=C_{\overline{\Delta \nu_{1}^{*}} ; \overline{\Delta \nu_{2}^{*}}}=P_{\frac{\nu}{\Delta \nu_{1}^{*}}}\left(\phi_{1}\right) P_{\overline{\Delta \nu_{2}^{*}}}^{\nu}\left(\phi_{2}\right) .
$$

The class operator for the complementary history in which the universe is at arbitrarily small volume at $\phi_{1}, \phi_{2}$, or both, is then

$$
\begin{aligned}
C_{\text {sing }}\left(\phi_{1}, \phi_{2}\right) & =\mathbb{1}-C_{\text {bounce }}\left(\phi_{1}, \phi_{2}\right) \\
& =C_{\Delta \nu_{1}^{*} ; \Delta \nu_{2}^{*}}+C_{\Delta \nu_{1}^{*} ; \overline{\Delta \nu_{2}^{*}}}+C_{\Delta \nu_{1}^{*} ; \Delta \nu_{2}^{*}} .
\end{aligned}
$$

By arguments essentially similar to those leading to (72) above, one shows that in the Wheeler-DeWitt quantum theory the branch wave functions corresponding to bouncing vs. singular cosmologies are

$$
\begin{gathered}
\left|\Psi_{\text {sing }}(\phi)\right\rangle=U\left(\phi-\phi_{o}\right) \lim _{\substack{\phi_{1} \rightarrow-\infty \\
\phi_{2} \rightarrow+\infty}} C_{\text {sing }}^{\dagger}\left(\phi_{1}, \phi_{2}\right)|\Psi\rangle=|\Psi(\phi)\rangle \\
\left|\Psi_{\text {bounce }}(\phi)\right\rangle=U\left(\phi-\phi_{o}\right) \lim _{\substack{\phi_{1} \rightarrow-\infty \\
\phi_{2} \rightarrow+\infty}} C_{\text {bounce }}^{\dagger}\left(\phi_{1}, \phi_{2}\right)|\Psi\rangle=0 .
\end{gathered}
$$

Thus the alternative histories (bounce,singular) decohere in this limit, $d$ (bounce, singular $)=\left\langle\Psi_{\text {sing }} \mid \Psi_{\text {bounce }}\right\rangle=0$, and

$$
\begin{aligned}
p_{\text {sing }} & =\left\langle\Psi_{\text {sing }} \mid \Psi_{\text {sing }}\right\rangle \\
& =\langle\Psi \mid \Psi\rangle \\
& =1 .
\end{aligned}
$$

Wheeler-DeWitt quantum cosmological models are invariably singular, regardless of state, in spite of the potential promise of Fig. 2 that they sometimes might not be.

Similarly, in loop quantum cosmology one finds instead that

$$
\begin{aligned}
\left|\Psi_{\text {bounce }}\right\rangle & =C_{\text {bounce }}|\Psi\rangle \\
& =|\Psi\rangle
\end{aligned}
$$

while

$$
\begin{aligned}
\left|\Psi_{\text {sing }}\right\rangle & =C_{\text {sing }}|\Psi\rangle \\
& =0
\end{aligned}
$$


Again, these histories decohere, but now $p_{\text {bounce }}=1$, and the probability arbitrary loop quantum states are singular is 0 .

\section{Discussion}

We have here reviewed the formulation of a consistent histories approach to quantum theory for loop quantum cosmology, showing how the framework provides the structure necessary - the decoherence functional - to enable the theory to make consistent quantum predictions in the absence of measurements, external observers, or other similar apparatus typically required in quantum theory before one can make definite predictions. We have illustrated the application of the framework in showing how loop quantum cosmologies are non-singular for generic states, contrasting that striking result with the inevitable singularity of the Wheeler-DeWitt quantization of the same family of comological models. We have also made an effort to point to some other work on consistent histories formulations of quantum cosmological models, even though there was not sufficient space to review them here.

Discussion of a few important points is in order. It is no secret that while physicists generally agree on how to do quantum mechanics, the story is quite different when one has the temerity to inquire what it means. We wish to emphasize that it is not necessary to commit to any particular ontology for quantum mechanics to acknowledge the centrality of the role of interference among alternative outcomes in arriving at quantum predictions. Indeed, destruction of this interference is the fundamental technical role played by the classical idea of "measurement" in quantum theory via the postulated "collapse" of the wave function upon measurement of a property by an external agent. Consistent histories quantum theory supplies, more or less, the minimal additional structure one requires to provide quantum theory with an objective, observer independent, purely internal measure of this interference, that reproduces the predictions of ordinary measurement-based quantum theory in traditional "measurement situations", but also extends it to physical circumstances in which there is no meaningful notion of an "external observer", thus enabling quantum theory to make predictions concerning questions of fundamental physical interest such as in the early universe. In particular, we emphasize that apart from introducing the decoherence functional, the objective measure of interference derived from the quantum state (an object already present in the conventional theory), consistent histories quantum theory is still quantum theory, with all of the interpretational challenges that implies. ${ }^{\circ}$ It is neither the place nor our intention here to analyze the limitations of the consistent histories framework as a complete answer to the question, "What is the quantum mechanical account of reality?" It is our view that while the concepts of consistent histories - even supplemented by physical mechanisms and ideas such as environmental decoherence, envariance, and

\footnotetext{
o Some of the foundational questions the consistent histories framework does not by itself attempt to directly answer include the problem of outcomes, the basis problem, and the meaning of "probability" - among others.
} 
"quantum Darwinism"15,16,18,19 - are at best only a partial answer to this question, we nonetheless believe it likely they will play a role in an eventual picture.

We have just alluded to the fact that the consistent histories framework does not offer a fresh answer to the "true" meaning of the probabilities quantum theory supplies. Nonetheless, we take it as uncontroversial that the meaning of a probability that is unity or zero is not ambiguous - the theory predicts that thing either does, or does not, happen, with certainty. ${ }^{5,51-53}$ These are the definite predictions of a theory. It is for this reason we have emphasized the $|\phi| \rightarrow \infty$ limit in our assessment of the probabilities that Wheeler-DeWitt or loop quantum universes are (or are not) singular. It is in this limit that we are guaranteed that all Wheeler-DeWitt states are singular, and that all loop quantum states bounce, quasiclassical or not.

It may be natural to inquire whether one could have argued that WheelerDeWitt cosmologies are necessarily singular even for superposition states such as (73) by arguing that the amplitude $\left\langle\Psi_{L} \mid \Psi_{R}\right\rangle=0$, without invoking the consistent histories formulation, and in spite of the fact that the "single- $\phi$ " probability illustrated in Fig. 2 suggests otherwise. (Indeed, one finds such arguments in the classical literature on quantum cosmology..$^{50,54}$ ) This is tempting, and is certainly the thrust of the consistent histories calculation itself. However, doing so assumes that this amplitude may be interpreted as a probability for a sequence of events, and thereby glosses over one of the fundamental messages of quantum theory: quantum amplitudes do not represent quantum probabilities unless interference among the alternative outcomes vanishes. ${ }^{\mathrm{p}}$ Simply put, quantum mechanics says that some questions do not have physically meaningful answers. In ordinary laboratory applications of quantum mechanics, the act of gathering information about a system - "measurement" - supplies the physical mechanisms that destroy that interference. Amplitudes for outcomes which are measured do not interfere, and therefore may be interpreted as probabilities for those measured outcomes. If the measurement is not made, those amplitudes may not be consistently interpreted as probabilities for the unmeasured outcomes. The consistent histories perspective on quantum theory simply recognizes that amplitudes are not probabilities unless such interference vanishes, and supplies an objective measure of that interference that may be applied in the absence of laboratories and measurements in environments such as the very early universe, where there were certainly no observers present. Even so, physical mechanisms may exist which destroy interfence among possible alternatives, and thereby enable quantum theory to assign definite probabilities to those alternatives. Indeed, additional degrees of freedom may supply a resource which effectively "gathers information" (i.e. creates records ${ }^{3-5,7,19,26}$ ) about the physical alternatives of interest, leading to decoherence of those alternative his-

PIt may be worth reiterating that this obtains generally when one considers amplitudes for $s e$ quences of quantum events - such as which slit a particle passes through in a two slit apparatus. But such amplitudes for quantum histories are precisely the sort in which one is often interested in quantum cosmology, such as the amplitudes for a quantum bounce discussed in this review. ${ }^{20-22,24}$ 
tories and consequent ability to assign them meaningful probabilities. One would expect this to be the case in the actual physical universe which even in a globally homogeneous and isotropic cosmology carries both geometric and matter degrees of freedom which may imprint information about quantum alternatives and lead to decoherence. Matter density perturbations in the early universe are just such degrees of freedom, and a consistent histories analysis of such perturbations should lead to a coherent picture of the "quantum-to-classical" transition of inflationary perturbations. ${ }^{55}$

Thus, the methods described in this review provide the tools to tell a consistent quantum story of the predictions of quantum gravitational theories in the early universe. In forthcoming work these methods will be applied to provide a similar analysis of spin-foam loop quantum cosmological models. ${ }^{30}$

\section{Acknowledgments}

The author would like to thank P. Singh for teaching him loop quantum cosmology

and for a fruitful collaboration. Portions of this work were supported by a grant from FQXi, for which we thank the Institute.

\section{References}

1. R. B. Griffiths, Consistent quantum theory (Cambridge University Press, Cambridge, 2008).

2. R. Omnès, The interpretation of quantum mechanics (Princeton University Press, Princeton, 1994).

3. M. Gell-Mann and J. B. Hartle, Quantum mechanics in the light of quantum cosmology, in Proceedings of the 3rd international symposium on the foundations of quantum mechanics in the light of new technology, eds. S. Kobayashi, H. Ezawa, M. Murayama and S. Nomura (Physical Society of Japan, Tokyo, 1990) pp. 321-343.

4. M. Gell-Mann and J. B. Hartle, Quantum mechanics in the light of quantum cosmology, in Complexity, Entropy, and the Physics of Information, ed. W. Zurek, SFI Studies in the Sciences of Complexity, Vol. VII (Addison-Wesley, Reading, 1990) pp. 425-458.

5. J. B. Hartle, The quantum mechanics of cosmology in Coleman et al. ${ }^{56}$ pp. $65-157$.

6. J. B. Hartle, Spacetime quantum mechanics and the quantum mechanics of spacetime, in Gravitation and Quantizations, Proceedings of the 1992 Les Houches Summer School, eds. B. Julia and J. Zinn-Justin (North Holland, Amsterdam, 1995) pp. 285480.

7. J. J. Halliwell, Phys. Rev. D60 (1999) 105031, arXiv:quant-ph/9902008 [quant-ph].

8. J. J. Halliwell and J. Thorwart, Phys. Rev. D64 (2001) 124018, arXiv:gr-qc/0106095 [gr-qc].

9. J. J. Halliwell and J. Thorwart, Phys. Rev. D65 (2002) 104009, arXiv:gr-qc/0201070 [gr-qc].

10. J. J. Halliwell and P. Wallden, Phys. Rev. D73 (2006) 024011, arXiv:gr-qc/0509013 [gr-qc].

11. J. J. Halliwell, Phys. Rev. D80 (2009) 124032, arXiv:0909.2597 [gr-qc].

12. J. B. Hartle and D. Marolf, Phys. Rev. D56 (1997) 6247, arXiv:gr-qc/9703021 [gr-qc]. 
13. D. A. Craig and J. B. Hartle, Phys. Rev. D69 (June 2004) 123525, arXiv: gr-qc/0309117v3 [gr-qc].

14. C. Anastopoulos and K. Savvidou, Class. Quant. Grav. 22 (2005) 1841, arXiv:gr-qc/0410131 [gr-qc].

15. E. Joos, H. D. Zeh, C. Kiefer, D. Giulini, J. Kupsch and I.-O. Stamatescu, Decoherence and the appearance of a classical world in quantum theory, second edn. (SpringerVerlag, Berlin, 2003).

16. M. Schlosshauer, Decoherence and the quantum-to-classical transition (SpringerVerlag, Berlin, 2007).

17. J. J. Halliwell, Phys. Rev. D39 (1989) 2912.

18. W. H. Zurek, Nature Physics 5 (2009) 181, arXiv:0903.5082 [quant-ph].

19. C. J. Riedel, W. H. Zurek and M. Zwolak, Phys. Rev. A93 (2016) 032126, arXiv:1312.0331 [quant-ph].

20. D. A. Craig and P. Singh, Found. Phys. 41 (2011) 371, arXiv:1001.4311 [gr-qc].

21. D. A. Craig and P. Singh, A consistent histories formulation of Wheeler-DeWitt quantum cosmology, in Quantum Theory: Reconsideration of Foundations - 5, ed. A. Krennikhov (American Institute of Physics, New York, 2010) pp. 275-282. Proceedings of the fifth Växjö conference on the foundations of quantum mechanics, 14-18 June 2009.

22. D. A. Craig and P. Singh, Phys. Rev. D82 (2010) 123526, arXiv:1006.3837 [gr-qc].

23. D. A. Craig and P. Singh, J. Phys.: Conf. Series 360 (2012) 012028.

24. D. A. Craig and P. Singh, Class. Quantum Grav. 30 (2013) 205008, arXiv:1306.6142 [gr-qc] .

25. D. A. Craig and P. Singh, Consistent probabilities in spin foam loop quantum cosmology (2016), in preparation.

26. M. Gell-Mann and J. B. Hartle, Phys. Rev. D47 (1993) 3345, arXiv:gr-qc/9210010 [gr-qc] .

27. L. Diosi, Phys. Rev. Lett. 92 (2004) 170401, arXiv:quant-ph/0310181 [quant-ph].

28. C. J. Isham, N. Linden and S. Schreckenberg, J. Math. Phys. 35 (1994) 6360.

29. D. A. Craig, The geometry of consistency: decohering histories in generalized quantum theory (1997), arXiv:quant-ph/9704031 [quant-ph].

30. D. A. Craig and P. Singh, Consistent probabilities in spin foam loop quantum cosmology (2016), in preparation.

31. D. P. Schroeren, Found. Phys. 43 (2013) 310, arXiv:1206.4553 [gr-qc].

32. A. Ashtekar and P. Singh, Class. Quantum Grav. 28 (2011) 213001, arXiv:1108.0893 [gr-qc] .

33. D. A. Craig, Class. Quantum Grav. 30 (2013) 035010, arXiv:1207.5601 [gr-qc].

34. A. Ashtekar, A. Corichi and P. Singh, Phys. Rev. D77 (2008) 024046, arXiv:0710.3565 [gr-qc].

35. A. Ashtekar, T. Pawlowski and P. Singh, Phys. Rev. D73 (2006) 124038, arXiv:gr-qc/0604013 [gr-qc].

36. A. Ashtekar, T. Pawlowski and P. Singh, Phys. Rev. D74 (2006) 084003, arXiv:gr-qc/0607039 [gr-qc].

37. P. Diener, B. Gupt and P. Singh, Class. Quantum Grav. 31 (2014) 105015, arXiv: 1402.6613 [gr-qc].

38. P. Diener, B. Gupt, M. Megevand and P. Singh, Class. Quantum Grav. 31 (2014) 165006, arXiv:1406.1486 [gr-qc].

39. A. Ashtekar, M. Campiglia and A. Henderson, Phys. Lett. B681 (2009) 347.

40. A. Ashtekar, M. Campiglia and A. Henderson, Class. Quantum Grav. 27 (2010) 135020, arXiv: 1001.5147v2 [gr-qc].

41. A. Ashtekar, M. Campiglia and A. Henderson, Phys. Rev. D82 (2010) 124043, 
arXiv:1011.1024 [gr-qc].

42. P. Singh, Phys. Rev. D73 (2006) 063508, arXiv:gr-qc/0603043 [gr-qc].

43. V. Taveras, Phys. Rev. D78 (2008) 064072, arXiv:0807.3325 [gr-qc].

44. A. Henderson, C. Rovelli, F. Vidotto and E. Wilson-Ewing, Class. Quantum Grav. 28 (2011) 025003, arXiv: 1010.0502v2 [gr-qc].

45. D. A. Craig, Branch wave functions for quasi-classical homogeneous universes, in Proceedings of the Eleventh Marcel Grossmann Meeting on General Relativity and Relativistic Astrophysics, ed. R. Ruffini (World Scientific, Singapore, 2007) pp. 1884-1886.

46. D. A. Craig and P. Singh, The vertex expansion in the consistent histories formulation of spin foam loop quantum cosmology, in Proceedings of the Fourteenth Marcel Grossmann Meeting on General Relativity and Relativistic Astrophysics, ed. R. Ruffini (World Scientific, Singapore, 2016) to appear.

47. D. A. Craig and P. Singh, Class. Quantum Grav. (2016) to appear.

48. F. Falciano, R. Pereira, N. Pinto-Neto and E. S. Santini, Phys. Rev. D86 (2012) 063504, arXiv:1206.4021 [gr-qc].

49. N. Pinto-Neto and J. Fabris, Class. Quantum Grav. 30 (2013) 143001, arXiv:1306.0820 [gr-qc].

50. J. J. Halliwell, Introductory lectures on quantum cosmology in Coleman et al. ${ }^{56} \mathrm{pp}$. 159-243.

51. J. B. Hartle, Quantum cosmology, in Highlights in gravitation and cosmology, eds. B. Iyer, A. Kembhavi, J. V. Narlikar and C. Vishveshwara (Cambridge University Press, Cambridge, 1988)

52. R. Sorkin, Mod. Phys. Lett. A9 (1994) 3119, arXiv:gr-qc/9401003 [gr-qc].

53. R. Sorkin, Quantum measure theory and its interpretation, in Quantum-classical correspondence: Proceedings of the 4th Drexel Symposium on Quantum Nonintegrability, eds. D. Feng and B.-L. Hu (International Press, Cambridge, Massachusetts, 1997) pp. 229-251.

54. C. Kiefer, Quantum gravity, third edn. (Oxford University Press, Oxford, 2012).

55. D. A. Craig and P. Singh, The quantum-to-classical transition of perturbations in quantum cosmology (2017), in preparation.

56. S. Coleman, J. B. Hartle, T. Piran and S. Weinberg (eds.), Quantum cosmology and baby universes: Proceedings of the 1989 Jerusalem Winter School for Theoretical Physics (World Scientific, Singapore, 1991). 